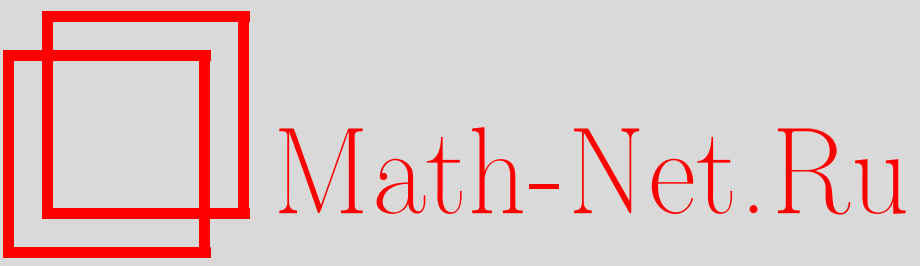

В. Г. Кановей, Топологии, порожденные эффективно суслинскими множествами, и их приложения в дескриптивной теории множеств, УМH, 1996, том 51, выпуск 3, 17-52

DOI: https://doi.org/10.4213/rm968

Использование Общероссийского математического портала Math-Net.Ru подразумевает, что вы прочитали и согласны с пользовательским соглашением

http: //www . mathnet.ru/rus/agreement

Параметры загрузки :

IP : 54.197 .217 .227

26 апреля 2023 г., 02:27:28 


\title{
ТОПОЛОГИИ, ПОРОЖДЕННЫЕ ЭФФЕКТИВНО СУСЛИНСКИМИ МНОЖЕСТВАМИ, И ИХ ПРИЛОЖЕНИЯ В ДЕСКРИПТИВНОЙ ТЕОРИИ МНОЖЕСТВ
}

\author{
В. Г. КАНОВЕЙ
}

СОДЕРЖАНИЕ

Введение . . . . . . . . . . . . . . .

1. Об эффективной дескриптивной теории множеств $\ldots \ldots \ldots \ldots \ldots \ldots \ldots \ldots . . . \ldots$

2. Топологии, порожденные эффективно суслинскими множествами .... 24

3. Первое приложение: ко-суслинские отношения эквивалентности ..... 30

4. Классификация борелевских отношений эквивалентности ........... 35

5. Расшепление плоских борелевских множеств .................... 44

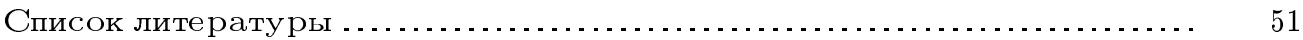

\section{Предисловие}

В 1994 году математическая общественность отметила 100-летие со дня рождения Михаила Суслина, российского математика большого таланта и трагической судьбы. Суслин родился в Саратовской губернии 15 (3) ноября 1894 г., окончил в 1917 году Московский университет, некоторое время преподавал в Иваново-Вознесенском политехническом институте - и умер от тифа в октябре 1919 г. на своей родине. ${ }^{1}$

Получив летом 1994 г. на Конференции памяти Суслина в Саратове почетное предложение написать статью в "Успехи математических наук" об одном из современных разделов в основаниях математики, где идеи Суслина находят новое воплошение, ав-

Работа выполнена при поддержке фондов DFG, AMS и ISF.

${ }^{1}$ Статья В. Игошина в этом выпуске дает материал по биографии Суслина. Статья [7] предлагает развернутьй анализ математической деятельности Суслина. В. М. Тихомиров освещает в [6] важные исторические и математические моменты, связанные с деятельностью группы молодых московских математиков под руководством Н. Н. Лузина, к которой принадлежал и Суслин. Все это делает излишним более обширные экскурсы в эти темы в предлагаемой статье. 
тор не долго колебался, выбрав темой суслинские множества. ${ }^{2}$ Также не вызвал особых колебаний и выбор конкретного направления: это топологии, порожденные эффективно суслинскими множествами, теоретико-множественный аппарат, возникший в конце 70-х годов как синтез некоторых идей топологии полных метрических и близких к ним пространств и “эффективной” дескриптивной теории множеств и ставший в настоящее время, после ряда замечательных успехов, едва ли не самым популярным методом в дескриптивной теории множеств. Характерно, например, что один из двух главных лекционных курсов на международной конференции топологов в Амстердаме (август 1994) был посвящен в значительной мере приложениям этого метода в топологии и дескриптивной теории множеств. ${ }^{3}$

Некоторые колебания вызвал лишш выбор стиля статьи: шшрокий обзор результатов или более узкая статья, представляющая в той или иной степени структуру доказательств. Автор избрал второй вариант, считая что такое изложение метода ${ }^{4}$ в доступном журнале высшего ранга будет в большей мере способствовать ознакомлению отечественных математиков с приложениями топологии эффективно суслинских множеств.

Автору приятно упомянуть помощь В. М. Тихомирова и В. А. Успенского в написании и опубликовании этой статьи.

\section{Введение}

Обычно, чем проще множества мы рассматриваем, тем легче доказьвать их свойства. Допустим, что то или иное интересующее нас свойство доказано для всех "простых", скажем, открытых множеств вещественной прямой $\mathbb{R}$, бэровского пространства $\mathscr{N}=\omega^{\omega}$ или другого польского ${ }^{5}$ пространства, но желательно распространить этот результат на более сложные множества, к примеру, борелевские или суслинские. Конечно, как правило, это требует и более сложной техники. Имеется однако и другой подход.

Именно, мы изменим саму топологию пространства, с тем чтобы те более сложные множества, которые нас интересовали, стали, например, открытыми в новой топологии. Естественно, при этом необходимо проследить, какую форму примет то свойство, которое мы хотим доказать с точки зрения новой топологии, а также быть уверенными, что новая топология достаточно хороша, чтобы позволить те рассуждения в отношении "простых" множеств, которые позволяла старая польская топология.

Этот подход оказался весьма успешно реализованньм в дескриптивной теории множеств при помощи топологии, идея которой принадлежит Ганди, но первое замеча-

\footnotetext{
${ }^{2}$ При жизни Суслина под его именем была опубликована всего одна математическая работа - это заметка [3] в Comptes Rendus, одном из наиболее авторитетных журналов того времени. Именно ею были введены в математику суслинские множества, которые сам Суслин назвал множеств ами (А). Они также известны под названиями: $A$-множества, или множества класса $\Sigma_{1}^{1}$.

${ }^{3}$ Материал курса, прочитанного А. С. Кекрисом, изложен в его статье [16].

${ }^{4}$ Вообще, кажется, первое на русском язьке.

${ }^{5}$ Полного метрического сепарабельного; часто с дополнительным условием отсутствия изолированных точек: совершенное польское пространство.
} 
тельное приложение 6 было получено Харрингтоном. Это топология, базой которой служат $\Sigma_{1}^{1}$-множества, или, в более общем плане, множества класса $\Sigma_{1}^{1}(p)$, где $p \in \mathscr{N}$ фиксировано (свойства топологии практически не зависят от выбора $p$ ).

Терминологическое пояснение. Класс $\Sigma_{1}^{1}$ образуют множества, которые можно получить $A$-операцией над әффективными (в данном случае - вычислимыми) семействами замкнутых множеств рассматриваемого пространства, в связи с чем множества из $\Sigma_{1}^{1}$ уместно назвать эффективно суслинскими. В более общем смысле, это название распространяется на множества любого класса $\Sigma_{1}^{1}(p)$, коль скоро точка $p$ бэровского пространства фиксирована.

Мы избрали три теоремы (все они относятся к числу наиболее важных из достаточно недавних результатов в дескриптивной теории множеств) для демонстрации приложений топологий, порожденных эффективно суслинскими множествами: это теорема Сильвера о числе классов эквивалентности ко-суслинских (или, что то же самое, $\boldsymbol{\Pi}_{1}^{1}$ ) отношений эквивалентности (раздел 3), теорема Харрингтона-Кекриса-Луво о дихотомии Глимма-Эффроса для борелевских отношений эквивалентности (раздел 4) и теорема Луво о представлении плоских борелевских множеств с вертикальными сечениями определенного аддитивного борелевского класса в виде счетной суммы борелевских множеств с сечениями меньших классов (раздел 5).

Но перед этим раздел 2 содержит введение в рассматриваемый класс топологий, а раздел 1 представляет главные определения и некоторые принципиальные теоремы эффективной дескриптивной теории множеств, ${ }^{7}$ используемые в изложении.

Такова в обших чертах организация статьи.

Выбор в отношении характера статьи повлек за собой определенноепренебрежение приложениями метода, в особенности связанными с теорией меры и борелевскими группами преобразований, в пользу материала, относящегося к “чистой" дескриптивной теории множеств, хотя эти как бы прикладные задачи во многом инспирировали разработку метода как такового. Этот круг вопросов освешен в статье А. С. Кекриca $[16]$.

\section{1. Об эффективной дескриптивной теории множеств}

Этот раздел дает введение в терминологию и краткий обзор некоторых важных теорем эффективной дескриптивной теории, в принципе достаточньй, скажем, для тополога, работавшего с дескриптивной теорией и имеюшего некоторое представление о теории рекурсии, чтобы следовать изложению.

\footnotetext{
${ }^{6}$ Новое доказательство теоремы Сильвера о ко-суслинских отношениях эквивалентности, см. ниже раздел 3. Следует отметить, что ни Ганди, ни Харрингтон не опубликовали материалы своих пионерских исследований на эту тему; их приоритет признан и известен из работ последователей.

${ }^{7}$ Разумеется, эта область, как и, наверное, любая область математики включает существенное "фольклорное" знание, иногда трудно поддающееся компактной формализации и конкретно здесь потребовавшее бы больше места, чем данная статья может предоставить. Поэтому для понимания технических деталей изложения было бы полезным предварительное знакомство с эффективной теорией в рамках, скажем, статьи Мартина [5].
} 
1.1. Пространства и множества. Рассматриваются множества пространств вида $\mathscr{X}=\omega^{k} \times \mathscr{N}^{m}$, где $k, m$ - натуральные числа, $\omega=\{0,1,2, \ldots\}$ - множество натуральных чисел, $\mathscr{N}=\omega^{\omega}-$ пространство Бәра. Точки этих пространств (в частности, $\mathcal{N}$ ) будут обозначаться буквами $p, q, x, y, z$, иногда буквами $a, b, c$, натуральные числа - буквами $i, j, k, l, m, n$.

Иногда бывает удобно рассматривать множества $P \subseteq \mathscr{X}=\omega^{k} \times \mathscr{N}^{m}$ как $k+m$-арные отношения; так, $P\left(n_{1}, \ldots, n_{k}, x_{1}, \ldots, x_{m}\right)$ выражает то же, что и $\left\{n_{1}, \ldots, n_{k}, x_{1}, \ldots, x_{m}\right\} \in P$. Этот стиль не будет использоваться в случае, когда может возникнуть путаника с функцией, а также когда $k+m=1$.

Зафиксировав на $\omega$ дискретную метрику (например, $\delta(k, l)=1$ при $k \neq l$ ), а на $\mathscr{N}$ - бэровскую метрику $\rho(x, y)=n^{-1}$, где $n=\max \left\{n^{\prime}: x \uparrow n^{\prime}=y \uparrow n^{\prime}\right\}$ при $x \neq$ $y \in \mathscr{N}$, мы делаем рассматриваемые пространства $\mathscr{X}=\omega^{k} \times \mathscr{N}^{m}$ сепарабельными полными метрическими; эта метрика и порожденная ею топология будут называться ниже польскими метрикой и топологией.

1.2. Классические иерархии. Борелевская иерархия классов $\boldsymbol{\Sigma}_{\xi}^{0}, \boldsymbol{\Pi}_{\xi}^{0}, \boldsymbol{\Delta}_{\xi}^{0}$, $1 \leqslant \xi<\omega_{1}$, определяется трансфинитной индукцией по $\xi$ :

- $\boldsymbol{\Sigma}_{1}^{0}=$ все открытые множества данного пространства;

- $\boldsymbol{\Pi}_{\xi}^{0}=$ дополнения множеств из $\boldsymbol{\Sigma}_{\xi}^{0} ; \boldsymbol{\Delta}_{\xi}^{0}=\boldsymbol{\Sigma}_{\xi}^{0} \cap \boldsymbol{\Pi}_{\xi}^{0}$;

- $\boldsymbol{\Sigma}_{\xi}^{0}=$ счетные объединения множеств из $\boldsymbol{\Pi}_{\eta}^{0}, 1 \leqslant \eta<\xi$ при $\xi>1$.

В частности, $\Pi_{1}^{0}=$ замкнутые множества, $\boldsymbol{\Sigma}_{2}^{0}=\mathbb{F}_{\sigma}, \Pi_{2}^{0}=\mathbb{G}_{\delta}$, и т. д.

Будет рассматриваться и начальньй уровень проективной иерархии:

- $\boldsymbol{\Sigma}_{1}^{1}$ - совокупность всех проекций $\boldsymbol{\Pi}_{1}^{0}$-множеств. Иными словами, множество $X \subseteq \mathscr{X}$ принадлежит $\boldsymbol{\Sigma}_{1}^{1}$, если найдется (замкнутое) $\boldsymbol{\Pi}_{1}^{0}$-множество $P \subseteq$ $\mathscr{X} \times \mathscr{N}$ такое, что $X=\{x \in \mathscr{X}: \exists y \in \mathscr{N} P(x, y)\}$.

- $\boldsymbol{\Pi}_{1}^{1}=$ дополнения множеств из $\boldsymbol{\Sigma}_{1}^{1} ; \boldsymbol{\Delta}_{1}^{1}=\boldsymbol{\Sigma}_{1}^{1} \cap \boldsymbol{\Pi}_{1}^{1}$.

По теореме Суслина, $\boldsymbol{\Delta}_{1}^{1}=$ все борелевские множества.

1.3. Нумерация баз. Все рассматриваемые пространства имеют счетную базу; таким образом, коль скоро задано перечисление всех множеств базы, каждое открытое множество будет определяться некоторым множеством натуральных чисел - номеров множеств базы. Идея эффективной теории состоит в том, чтобы рассматривать лиш вычислимые объединения базовых множеств.

Вопрос о том, как все это зависит от выбора конкретного перечисления множеств базы, здесь не представляет интереса; мы просто фиксируем одно конкретное перечисление для каждого пространства.

Натуральные числа. Мы полагаем $B_{n+1}[\omega]=\{n\}$ и отдельно $B_{0}[\omega]=\varnothing$ (пустое множество).

Пространство Бэра. Здесь придется немного повозиться.

Пусть $\ulcorner k, l\urcorner=2^{k}(2 l+1)-1$ для $k, l \in \omega$ (арифометическая "пара"). Нумерация $\omega^{<\omega}=\left\{s_{n}: n \in \omega\right\}$ множества $\omega^{<\omega}$ всех конечных последовательностей натуральных чисел определяется так: $s_{0}=s_{1}=\Lambda$ (пустая последовательность), и, если $n=\ulcorner k, l\urcorner \geqslant 2$, то $s_{n}=s_{k} \wedge l$. Мы полагаем $B_{n+1}[\mathcal{N}]=\left\{x \in \mathscr{N}: s_{n} \subset x\right\}$ и отдельно $B_{0}[\mathcal{N}]=\varnothing$. 
Общий случай: пространство $\mathscr{X}=\omega^{l} \times \mathscr{N}^{m}$. Пусть $n=\pi_{1}^{k_{1}} \cdots \pi_{l+m}^{k_{n+m}}$, где $\pi_{i}$ есть $i$-е простое число. Полагаем

$$
B_{n}[\mathscr{X}]=B_{k_{1}}[\omega] \times \cdots \times B_{k_{l}}[\omega] \times B_{k_{l+1}}[\mathcal{N}] \times \cdots \times B_{k_{l+m}}[\mathscr{N}]
$$

при $n \geqslant 1$ и отдельно $B_{0}[\mathscr{X}]=\varnothing\left(\right.$ это совпадает с $\left.B_{1}[\mathscr{X}]\right)$.

\section{4. Эффективная иерархия.}

- $\Sigma_{1}^{0}$ (в пространстве $\mathscr{X}$ ) есть класс всех множеств вида $X=\bigcup_{n} B_{f(n)}[\mathscr{X}]$, где $f \in \mathscr{N}$ - вычислимая функция.

- Пусть $p \in \mathscr{N} . \Sigma_{1}^{0}(p)$ есть класс всех множеств вида $X=\bigcup_{n} B_{f(n)}[\mathscr{X}]$, где $f \in \mathscr{N}$ - функция, вычислимая относительно $p$.

(Можно считать, что вычислимая функция $f: \omega \rightarrow \omega$-это та, для вычисления которой имеется компьютерная программа. Если речь идет о более широком понятии программы, которое включает обрашение к значениям $p(n)$ некоторой данной функции $p$ (которая сама может не быть вычислимой), то мы получаем вычислимость относительно $p$. Вообще, предполагается некоторое знакомство с этими понятиями.)

- $\Pi_{1}^{0}=$ дополнения множеств из $\Sigma_{1}^{0} ; \Delta_{1}^{0}=\Sigma_{1}^{0} \cap \Pi_{1}^{0}$.

- $\Sigma_{1}^{1}=$ проекции $\Pi_{1}^{0}$-множеств.

- $\Pi_{1}^{1}=$ дополнения множеств из $\Sigma_{1}^{1} ; \Delta_{1}^{1}=\Sigma_{1}^{1} \cap \Pi_{1}^{1}$.

- Классы $\Pi_{1}^{0}(p), \Delta_{1}^{0}(p), \Sigma_{1}^{1}(p), \Pi_{1}^{1}(p), \Delta_{1}^{1}(p)$ определяются аналогично.

Начальные классы $\Sigma_{1}^{0}$ и $\Sigma_{1}^{0}(p)$ при любом $p$ и, следовательно, все остальные классы $\Gamma_{i}^{1}, \Gamma_{i}^{1}(p){ }^{8}$ содержат лиш счетное число множеств, в отличие от их "классических" прототипов $\Gamma_{\ldots}^{i}$.

Мы умьшленно не даем здесь определение классов $\Gamma_{\xi}^{0}$ для $2 \leqslant \xi<\omega_{1}$; это непростой вопрос ${ }^{9}$ он будет рассмотрен ниже в разделе 5 .

Эта классификация по определению распространена и на множества натуральных чисел и вообще подмножества пространств $\omega^{k}$; в частности, поскольку каждая точка $x \in \mathscr{N}$ есть функция из $\mathcal{N}$ в $\mathscr{N}$, т.е. подмножество $\omega^{2}$ (как график), это придает смысл выражениям типа $x \in \Sigma_{1}^{1}(p)$, где $x$ и $p$ принадлежат $\mathcal{N}$.

1.5. Взаимоотношения между классами. При любом $p$ и $i=0,1$ мы имеем $\Sigma_{1}^{i}(p) \nsubseteq \Pi_{1}^{i}(p) ;$ с другой стороны, справедливо $\Sigma_{1}^{0}(p) \cup \Pi_{1}^{0}(p) \subseteq \Delta_{1}^{1}(p)$.

Далее, иерархия монотонна по $p$ в том смысле, что если $q \in \Delta_{1}^{0}(p)$, то $\Gamma_{1}^{i}(q) \subseteq$ $\Gamma_{1}^{i}(p)$. Классы $\Gamma_{1}^{i}$ тождественны $\Gamma_{1}^{i}(p)$ при $p \in \Delta_{1}^{0}$; в частности, $\Gamma_{1}^{i}=\Gamma_{1}^{i}(\mathbf{0})$, где точка $\mathbf{0} \in \mathcal{N}$ задана условием $\mathbf{0}(k)=0$ для всех $k$.

Связь с классической иерархией: $\boldsymbol{\Gamma}_{1}^{i}=\bigcup_{p \in \mathcal{N}} \Gamma_{1}^{i}(p)$.

Подстановка параметров: если $P(x, y, z, \ldots)$ - отношение класса $\Gamma_{1}^{1}(p)$ и $x_{0} \in \mathscr{N}$ принадлежит $\Delta_{1}^{1}(p)$, то отношение $Q(y, z, \ldots)$, определенное как $P\left(x_{0}, y, z, \ldots\right)$, также входит в $\Gamma_{1}^{1}(p)$. (Как обычно, $\Gamma=\Sigma, \Pi$ или $\Delta$.)

\footnotetext{
${ }^{8}$ Через $\Gamma$ и $\boldsymbol{\Gamma}$ принято обозначать любой из классифокикаторов $\Sigma, \Pi, \Delta$ или, соответственно, $\boldsymbol{\Sigma}, \boldsymbol{\Pi}, \Delta$

${ }^{9}$ Если не считать относительно небольших, например, конечных, значений $\xi$, когда определение достаточно элементарно.
} 
Имеются простые правила (см., например, книгу Шенфилда [8, гл. 7.8]), позволяющие оценить класс множества, полученного действием определенных операций на множества известных классов. Эти правила удобно представлять, трактуя множества как отношения, см. раздел 1.1, когда становится возможным использовать язык логики.

Отрицание. Отрицание $\Sigma_{1}^{i}(p)$-отношения есть отношение класса $\Pi_{1}^{i}(p)$, и наоборот. На языке множеств, этим выражается тот факт, что множества из $\Sigma_{1}^{i}(p)$ и $\Pi_{1}^{i}(p)$ взаимно дополнительны.

Конъюнкция и дизъюнкция. Все классы, определенные в разделе 1.4 , замкнуты относительно этих операций (в конечной форме).

Кванторы типа $\omega$. Классы $\Sigma_{1}^{0}(p)$ и все $\Gamma_{1}^{1}(p), \Gamma=\Sigma, \Pi, \Delta$, замкнуты относительно квантора $\exists n \in \omega$. Иными словами, если $P(n, x, y, \ldots)$ есть, скажем, $\Sigma_{1}^{0}(p)$-отношение, то отношение $Q(x, y, \ldots) \longleftrightarrow \exists n \in \omega P(n, x, y, \ldots)$ принадлежит тому же класcy $\Sigma_{1}^{0}(p)$.

Классы $\Pi_{1}^{0}(p)$ и все $\Gamma_{1}^{1}(p)$ замкнуты относительно $\forall z \in \omega$.

Кванторы типа $\mathcal{N}$. Классы $\Sigma_{1}^{0}(p)$ и $\Sigma_{1}^{1}(p)$ замкнуты относительно квантора $\exists z \in \mathscr{N}$. Классы $\Pi_{1}^{0}(p)$ и $\Pi_{1}^{1}(p)$ замкнуты относительно $\forall z \in \mathscr{N}$.

Эти правила будут систематически использоваться в изложении.

1.6. Униформизация, редукция, отделимость.

Униформизация. Пусть $P \subseteq \mathscr{X} \times \mathscr{Y}$. Говорят, что множество $Q \subseteq P$ униформизует $P$, если, во-первых, проекция $Q$ на $\mathscr{X}$ совпадает с проекцией $P$ на $\mathscr{X}$, т.е. формально, для любого $x \in \mathscr{X}$, если $\exists y P(x, y)$, то $\exists y Q(x, y)$, и, во-вторых, $Q$ - униформное множество, т.е. множество, пересекаемое каждой “вертикальной прямой" $x=x_{0}$ не более чем в одной точке. Униформное множество можно понимать как график функции, отображаюшей подмножество $\mathscr{X}$ в $\mathscr{Y}$.

Принцип 1 (Новиков-Кондо-Аддисон, см. [8, гл. 7.11]). Пусть $P \subseteq \mathscr{X} \times \mathscr{Y}$ есть $\Pi_{1}^{1}(p)$-множество, $p \in \mathscr{N}$. Найдется $\Pi_{1}^{1}(p)$-множество $Q \subseteq P$, униформизуюuеe $P$.

Известны важные случаи, когда униформизация может быть достигнута при помоши множества класса $\Delta_{1}^{1}$. Мы приведем один из них, представляющий особый интерес.

СлеДСТВИЕ 2. Если, в условиях принщипа 1, $\mathscr{Y}=\omega, a X=\{x: \exists y P(x, y)\}$ (т.е. проекиия $P$ на $\mathscr{X})$ есть $\Sigma_{1}^{1}(p)$-множество, то униформизующее множество $Q$ может бить выбрано в классе $\Delta_{1}^{1}(p)$.

ДокаЗАТЕльство. Возьмем произвольное $\Pi_{1}^{1}(p)$-множество $Q \subseteq P$, униформизуюшее $P$. Тогда $Q$ автоматически принадлежит и $\Sigma_{1}^{1}(p)$, поскольку

$$
Q(x, k) \longleftrightarrow x \in X \& \forall k^{\prime} \neq k \neg Q\left(x, k^{\prime}\right)
$$

Редукция и отделимость. Говорят, что пара множеств $A^{\prime}, B^{\prime}$ редуцирует пару $A, B$, когда $A^{\prime} \subseteq A, B^{\prime} \subseteq B, A^{\prime} \cap B^{\prime}=\varnothing$, но $A^{\prime} \cup B^{\prime}=A \cup B$. Говорят, что множество $C$ отделяет $A$ от $B$, когда $\mathrm{A} \subseteq C$ и $B \cap C=\varnothing$. 
СЛЕДСТВиЕ 3 [Редукция и отделимость]. Пусть $p \in \mathscr{N}$. Любая пара $\Pi_{1}^{1}(p)$-множеств $A, B$ (в одном пространстве) может быть редуцирована парой $\Pi_{1}^{1}(p)$-множеств. Если $A$ и $B$ - непересекающиеся $\Sigma_{1}^{1}(p)$-множества, то имеется отделятощее $\Delta_{1}^{1}(p)$-множество.

ДокаЗАТельСтво. Множество $P=(A \times\{0\}) \cup(B \times\{1\})$ принадлежит $\Pi_{1}^{1}(p)$. Униформизуем $P$ множеством $Q \subseteq P$ из $\Pi_{1}^{1}(p)$; тогда $A^{\prime}=\{x: Q(x, 0)\}$ и $B^{\prime}=$ $\{x: Q(x, 1)\}$ будут $\Pi_{1}^{1}(p)$-множествами, что доказывает редукцию. Чтобы получить отделимость, достаточно произвести редукцию дополнительных множеств.

1.7. Нумерация классов $\Delta_{1}^{1}(p)$. Напомним, что в каждом классе $\Delta_{1}^{1}(p)$ имеется лишь счетное число множеств. Одним из наиболее важных технических средств эффективной теории является существование особых нумераций для этих классов.

Принцип 4 [Часть 1: нумерация $\Delta_{1}^{1}$-множеств]. Существуют $\Pi_{1}^{1}$-множество $\mathbf{W} \subseteq \mathscr{N} \times \omega$ и индексированное семейство $\left\langle\mathbf{D}_{n}(p):\langle p, n\rangle \in \mathbf{W}\right\rangle$ множеств $\mathbf{D}_{n}(p) \subseteq \mathscr{N}$ maкuе, что

(i) для любого $p \in \mathcal{N}$ множество $\left\{\mathbf{D}_{n}(p):\langle p, n\rangle \in \mathbf{W}\right\}$ тождественно совокупности всех $\Delta_{1}^{1}(p)$-множеств $X \subseteq \mathcal{N}$;

(ii) следующие множсества принадлежат $\Pi_{1}^{1}$ :

$$
\left\{\langle p, n, x\rangle: \mathbf{W}(p, n) \& x \in \mathbf{D}_{n}(p)\right\} \quad u \quad\left\{\langle p, n, x\rangle: \mathbf{W}(p, n) \& x \notin \mathbf{D}_{n}(p)\right\} .
$$

[Часть 2: нумерация $\Delta_{1}^{1}$-точек] Существуют $\Pi_{1}^{1}$-множество $\mathbf{w} \subseteq \mathscr{N} \times \omega u$ индексированное семейство $\left\langle\mathbf{d}_{n}(p):\langle p, n\rangle \in \mathbf{w}\right\rangle$ точек $\mathbf{d}_{n}(p) \in \mathscr{N}$ такие, что

(iii) для любого $p \in \mathscr{N}$ множество $\left\{\mathbf{d}_{n}(p):\langle p, n\rangle \in \mathbf{w}\right\}$ тождественно совокупности всех $\Delta_{1}^{1}(p)$-точек $x \in \mathscr{N}$;

(iv) следующие множества принадлежат $\Pi_{1}^{1}$ :

$$
\left\{\left\langle p, n, \mathbf{d}_{n}(p)\right\rangle:\langle p, n\rangle \in \mathbf{w}\right\} \quad u \quad\left\{\langle p, n, x\rangle:\langle p, n\rangle \in \mathbf{w} \& x \neq \mathbf{d}_{n}(p)\right\} .
$$

Итак, при любом $p \in \mathscr{N}$ мы имеем перечисление семейства всех $\Delta_{1}^{1}(p)$-множеств $X \subseteq \mathscr{N}$ в виде $\left\{\mathbf{D}_{n}(p): n \in \mathbf{W}(p)\right\}$, где $\mathbf{W}(p)=\{n: \mathbf{W}(p, n)\}$ есть множество класса $\Pi_{1}^{1}(p)$, и аналогичное перечисление совокупности всех $\Delta_{1}^{1}(p)$-точек $x \in \mathscr{N}$. Обе эти нумерации обладают удобными характеристиками определимости, близкими к $\Delta_{1}^{1}(p)$-определимости. (Известно, что в действительности $\Delta_{1}^{1}(p)$-перечисление в этой ситуации невозможно.)

ДокАЗАТЕЛЬСТво. 10 Часть 1 . Используя технику построения универсальных множеств (см. [5, теорема 4.9]), можно построить пару $\Pi_{1}^{1}$-множеств $U, V \subseteq$ $\mathscr{N} \times \omega \times \mathscr{N}$, дважды универсальную в том смысле, что для любого $p \in \mathscr{N}$, если множества $X, Y \subseteq \mathscr{N}$ принадлежат $\Pi_{1}^{1}(p)$, то найдется $n \in \omega$ такое, что $X=U_{p n}=\{x: U(p, n, x)\}$ и $Y=V_{p n}=\{x: V(p, n, x)\}$.

\footnotetext{
${ }^{10}$ Мы даем эскиз доказательства, поскольку, во-первых, это действительно ключевой факт, а, во-вторых, трудно предложить разумную ссылку на источник на русском языке.
} 
Следствие 3 дает пару $\Pi_{1}^{1}$-множеств $U^{\prime} \subseteq U, V^{\prime} \subseteq V$, которая редуцирует пару $U, V$, в частности, $U^{\prime} \cap V^{\prime}=\varnothing$. Пусть $\mathbf{W}=\left\{\langle p, n\rangle: U_{p n}^{\prime} \cup V_{p n}^{\prime}=\mathscr{N}\right\}$ и $\mathbf{D}_{n}(p)=U_{p n}^{\prime}=\left\{x: U^{\prime}(p, n, x)\right\}$ при $\langle p, n\rangle \in \mathbf{W}$. Чтобы доказать, что второе из множеств, названных в требовании (ii), принадлежит $\Pi_{1}^{1}$, нужно воспользоваться тем обстоятельством, что при $\langle p, n\rangle \in \mathbf{W}$ множества $U_{p n}^{\prime}$ и $V_{p n}^{\prime}$ взаимно дополнительны.

Часть 2. Здесь несколько иные выкладки. Начнем с $\Pi_{1}^{1}$-множества $U \subseteq \mathscr{N} \times \omega \times \omega^{2}$, универсального в том смысле, что для любого $p \in \mathcal{N}$, если множество $X \subseteq \omega^{2}$ принадлежит $\Pi_{1}^{1}(p)$, то найдется натуральное $n$ такое, что $X=U_{p n}=\{\langle k, l\rangle: U(p, n, k, l)\}$. Униформизуем $U$ как подмножество $(\mathscr{N} \times \omega \times \omega) \times \omega$ множеством $V \subseteq U$ класса $\Pi_{1}^{1}$. Принципиальный момент состоит в том, что если $U_{p n}$ уже является функцией, определенной на $\omega$, т.е. точкой $\mathcal{N}$, то $V_{p n}=U_{p n}$. Теперь мы достигаем результата, полагая

$$
\mathbf{w}=\left\{\langle p, n\rangle: U_{p n} \in \mathscr{N}\right\}=\{\langle p, n\rangle: \forall k \exists ! l U(p, n, k, l)\}
$$

и $\mathbf{d}_{n}(p)=V_{p n}=U_{p n}$ при $\langle p, n\rangle \in \mathbf{w}$. Чтобы доказать, что множества из (iv) принадлежат $\Pi_{1}^{1}$, нужно иметь в виду, что при $\langle p, n\rangle \in \mathbf{w}$

$$
\begin{aligned}
x=\mathbf{d}_{n}(p) & \longleftrightarrow \forall k, l \in \omega[U(p, n, k, l) \rightarrow x(k)=l] \\
& \longleftrightarrow \forall k, l \in \omega[x(k)=l \rightarrow U(p, n, k, l)] .
\end{aligned}
$$

СЛЕДСТВИЕ 5. Пусть $R(x, p, \ldots)$ является $\Pi_{1}^{1}$-отношением. Тогда отношение $\exists x \in \Delta_{1}^{1}(p) R(x, p, \ldots)$ также принадлежит $\Pi_{1}^{1}$.

ДокАЗАТЕЛЬСТВО. Используя принцип 4, мы имеем

$$
\exists x \in \Delta_{1}^{1}(p) R(x, p, \ldots) \longleftrightarrow \exists n\left[\mathbf{w}(p, n) \& \forall x\left(x=\mathbf{d}_{n}(p) \rightarrow R(x, n, \ldots)\right)\right]
$$

Посмотрим на правую часть. Квантор $\exists n$ есть $\exists n \in \omega$, т.е. он не в счет. Остается заметить, что $\mathbf{w}(p, n)$ есть $\Pi_{1}^{1}$-отношение, а равенство $x=\mathbf{d}_{n}(p)$ может быть выражено $\Sigma_{1}^{1}$-формулой $\neg\left(x \neq \mathbf{d}_{n}(p)\right)$.

СЛЕДСТвИЕ 6. Множество $\Delta=\left\langle\langle p, x\rangle \in \mathscr{N}^{2}: x \in \Delta_{1}^{1}(p)\right\rangle$ принадлежит $\Pi_{1}^{1}$.

ДокАЗАТЕЛЬСТво. $\Delta(p, x) \longleftrightarrow \exists x^{\prime} \in \Delta_{1}^{1}(p)\left(x=x^{\prime}\right)$.

\section{2. Топологии, порожденные эффективно суслинскими множествами}

Теперь мы имеем терминологический базис для того, чтобы формально ввести это семейство топологий. ${ }^{11}$

\section{1. Топология.}

ОПРЕДЕЛЕНИЕ 7. $\mathscr{T ~ е с т ь ~ т о п о л о г и я , ~ б а з о и ̆ ~ к о т о р о и ̆ ~ я в л я ю т с я ~ в с е ~} \Sigma_{1}^{1}$-множества данного пространства. Аналогично, при $p \in \mathscr{N}, \mathscr{T}(p)$ есть топология, порожденная $\Sigma_{1}^{1}(p)$-множествами данного пространства.

\footnotetext{
${ }^{11}$ Значительная часть материала этого раздела заимствована из статей Мартина и Кекриса [20], Луво [18], Кекриса [16], Харрингтона, Кекриса и Луво [12] и книги Мэнсффилда и Уэйткампа [19], где можно найти более полную информацию.
} 
Это довольно необычные топологии. $\mathscr{T}(p)$ усиливает польскую топологию, так как все бэровские интервалы принадлежат $\Sigma_{1}^{1}(p)$. Однако $\mathscr{T}(p)$ делает открытыми и некоторые одноточечные множества; точнее, $\{x\}$ открыто в $\mathscr{T}(p)$, если $x \in \Delta_{1}^{1}(p)$. $\mathrm{C}$ другой стороны, $\mathscr{T}(p)$ имеет счетную базу.

Далее, эти топологии не являются польскими; действительно, простое свойство топологий, метризуемых полной метрикой, заключается в том, что каждое замкнутое множество есть $\mathbb{G}_{\delta}$. Рассмотрим произвольное $\Pi_{1}^{1}$-множество, не являюшееся $\Sigma_{1}^{1}$-множеством. (Сушествование таких множеств легко доказывается в рамках эффективной теории.) Это множество замкнуто в $\mathscr{T}(p)$ по определению, но оно не может быть $\mathbb{G}_{\delta}$, поскольку любое $\mathbb{G}_{\delta}$ в смысле $\mathscr{T}(p)$ есть $\Sigma_{1}^{1}$ в смысле польской топологии.

В то же время некоторые дериваты полноты польских пространств унаследуются топологиями $\mathscr{T}(p)$. В частности, мы увидим ниже (следствие 13$)$, что все они - бэровские.

Для доказательства этого свойства необходимо иметь способ построения убывающих последовательностей множеств с непустым пересечением в данной топологии, подобньй, скажем, тому, который дается полной метрикой. В классических изложениях темы (см. сноску 11) это делается при помоши игр Шоке. Мы используем другую технику, более близкую методам полных метрических пространств, но также рассмотрим и метод, основанньй на играх Шоке.

Следуя принятой практике, мы будем ниже рассматривать только топологию $\mathscr{T}$; однако все результаты верны для любой из топологий $\mathscr{T}(p)$, с соответствуюшими очевидньми изменениями.

2.2. Обеспечение непустоты пересечений. Следуюшее определение вводит структуру, которая достаточно успешно заменит полную метризуемость для топологии $\mathscr{T}$.

ОПРЕДЕЛЕНИЕ 8 . Совокупность семейств множеств $\mathscr{X}_{m}(m \in \omega)$ мы назовем польской сетью, если

(1) каждое $\mathscr{X}_{m}$ есть семейство непустых открытых подмножеств данного пространства;

(2) каждое $\mathscr{X}_{m}$ плотно в топологии: для любого непустого открытого множества $X$ сушествует $Y \in \mathscr{X}_{m}, Y \subseteq X$;

(3) компактность: если $X_{m} \in \mathscr{X}_{m}$ и $X_{\leqslant m}=\bigcap_{k \leqslant m} X_{k} \neq \varnothing$ для всех $m$, то пересечение $\bigcap_{m \in \omega} X_{m}$ содержит ровно одну точку.

Например, для $\mathscr{N}$ с обычной топологией мы определяем польскую сеть посредством $\mathscr{X}_{m}=\left\{\mathscr{N}_{s}: s \in \omega^{m}\right\}$, где $\mathscr{N}_{s}=\{x \in \mathscr{N}: s \subseteq x\}$ при $s \in \omega^{<\omega}$.

Лемма 9. Топология Т Тмеет польскую сеть, удовлетворяющую следующему дополнительному требованию "генеричности":

(4) Ко всякому $\Sigma_{1}^{1}$-множеству $A \subseteq \mathscr{N}$ найдется $m \in \omega$ такое, что любое $X \in \mathscr{X}_{m}$ удовлетворяет требованию: $X \subseteq A$ или $X \cap A=\varnothing$. 
ДокаЗАтельство. Рассмотрим пространство $\mathscr{N}^{1+\omega}=\mathscr{N} \times \mathscr{N}^{\omega}$. Точки $\vec{x} \in$ $\mathscr{N}^{1+\omega}$ удобно представлять в виде: $\vec{x}=\left\langle x, x_{0}, x_{1}, x_{2}, \ldots\right\rangle$. Для $P \subseteq \mathscr{N}^{1+\omega}$ положим $\operatorname{pr} P=\left\{x: \exists \vec{x}=\left\langle x, x_{0}, \ldots\right\rangle \in P\right\} \quad$ и $\operatorname{pr}_{n} P=\left\{x_{n}: \exists \vec{x}=\left\langle x, x_{0}, \ldots, x_{n}, \ldots\right\rangle \in P\right\}$

для всех $n$. Мы также полагаем $\vec{x} \uparrow \leqslant n=\left\langle x, x_{0}, x_{1}, \ldots, x_{n}\right\rangle$ для любой точки $\vec{x}=$

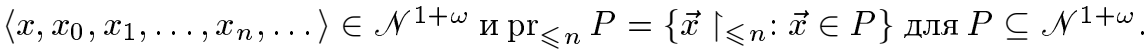

Определим $\mathscr{B}_{n}$ как семейство всех $\Pi_{1}^{0}$-множеств $B \subseteq \mathscr{N}^{1+\omega}$ вида

$$
B=\operatorname{pr}_{\leqslant n}^{-1}(B)=\left\{\vec{x} \in \mathscr{N}^{1+\omega}: \vec{x}\left\lceil\leqslant n \in B^{\prime}\right\}, \quad \text { где } B^{\prime} \subseteq \mathscr{N}^{1+n} \text { есть } \Pi_{1}^{0}\right. \text {-множество, }
$$

для каждого $n$. Понятно что $\mathscr{B}=\bigcup_{n} \mathscr{B}_{n}$ счетно; пусть $\mathscr{B}=\left\{\mathrm{B}^{m}: m \in \omega\right\}$, где любое $B \in \mathscr{B}$ имеет бесконечно много номеров $m$ таких, что $B=\mathrm{B}^{m}$.

Пусть $m \in \omega$. Мы вводим совокупность $\mathscr{X}_{m}$ всех непустых $\Sigma_{1}^{1}$-множеств $X=$ $\operatorname{pr} B \subseteq \mathscr{N}$, где (непустое) $B \in \mathscr{B}$ удовлетворяет следующим условиям:

(i) $\operatorname{diam} \operatorname{pr}_{k} B \leqslant m^{-1}$ для всех $k \leqslant m$ и diam pr $B \leqslant m^{-1} ;{ }^{12}$

(ii) либо $X \cap \operatorname{pr} \mathrm{B}^{m}=\varnothing$, либо $B \subseteq \mathrm{B}^{m}$.

Мы доказываем, что семейства $\mathscr{X}_{m}$ образуют польскую сеть. Для этого потребуется

ПРЕДЛОЖЕНИЕ 10. Пусть $Q \in \mathscr{B} u X \subseteq \operatorname{pr} Q$ есть непустое $\Sigma_{1}^{1}$-множество. Найдется множество $B \in \mathscr{B}, B \subseteq Q$, такое, ито $\operatorname{pr} B=X$.

ДоказАтельство. Имеется $\Pi_{1}^{0}$-множество $F \subseteq \mathscr{N}^{2}$, выполняющее $X=\operatorname{pr} F=$ $\{x: \exists y F(x, y)\}$. Пусть $Q \in \mathscr{B}_{n}$. Тогда $B=\left\{\vec{x} \in Q:\left\langle x, x_{n+1}\right\rangle \in F\right\}$ принадлежит $\mathscr{B}_{n+1}\left(\right.$ так как $\left.Q \in \mathscr{B}_{n}\right)$ и $\operatorname{pr} B=X$.

Мы возврашаемся к доказательству леммы.

Проверяем плотность $\mathscr{X}_{m}$ - условие (2) определения 8. Пусть $\Sigma_{1}^{1}$-множество $X \subseteq \mathscr{N}$ непусто; требуется найти $Y \in \mathscr{X}_{n}$ такое, что $Y \subseteq X$.

Случай 1: $X \cap \operatorname{prB}{ }^{m}=\varnothing$. Тогда $X=\operatorname{pr} Q$ для подходящего $Q \in \mathscr{B}_{0}$, поскольку $X \in \Sigma_{1}^{1}$. Пересекая $Q$ с “бэровским кубом" достаточно малого диаметра в $\mathscr{N}^{1+\omega}$, мы получаем непустое $\Pi_{1}^{0}$-множество $B \in \mathscr{B}, B \subseteq Q$, удовлетворяющее (i). Остается положить $Y=\operatorname{pr} B$.

Случай 2: $X^{\prime}=X \cap \operatorname{pr~B}^{m} \neq \varnothing$. Мы применяем предложение 10 к множествам $X^{\prime}$ и $Q=\mathrm{B}^{m}$. Имеем множество $Q^{\prime} \in \mathscr{B}, Q^{\prime} \subseteq Q$, такое, что $X^{\prime}=\operatorname{pr} Q^{\prime}$. Остается пересечь $Q^{\prime}$ с подходящим “кубом”, чтобы получить непустое множество $B \subseteq Q^{\prime}$, удовлетворяющее (i).

Проверяем компактность - условие (3). Итак, пусть $X_{m} \in \mathscr{X}_{m}$ и $\bigcap_{k \leqslant m} X_{k}$ непусто для каждого $m$; убедимся, что пересечение $\bigcap_{m} X_{m}$ имеет ровно одну точку. Будет достаточно показать, что, каково бы ни было $m=m_{0}$, сушествует бесконечная последовательность $m_{0}<m_{1}<m_{2}<\cdots$ такая, что $\bigcap_{n} X_{m_{n}}$ непусто; в самом деле, благодаря условию (i) каж дое непустое пересечение такого вида имеет ровно одну точку, и любые две точки, полученные этим способом, очевидно, равны одна другой.

\footnotetext{
${ }^{12}$ Диаметр $\operatorname{diam} X$ множества $X \subseteq \mathcal{N}$ понимается в смысле фиксированной полной метрики на $\mathscr{N}$, см. раздел 1.
} 
Итак, пусть $m_{0} \in \omega$. По индукции мы определяем номера $m_{n}$ и множества $P_{n} \in$ $\mathscr{B}_{m_{n}}$, удовлетворяюшие условиям $P_{n+1} \subseteq P_{n}, X_{m_{n}}=\operatorname{pr} \Pi_{n}$ и $\operatorname{diam~pr}_{k} P_{n} \leqslant m_{n}^{-1}$ для любого $k \leqslant m_{n}$. Тогда пересечение $\bigcap_{n} P_{n}$ непусто (каждое $P_{n}$ замкнуто в польской топологии пространства $\mathscr{N}^{1+\omega}$ и диаметры сходятся к нулю), т.е. пересечение $\bigcap_{n} X_{m_{n}}$ также непусто.

Поскольку $X_{m_{0}} \in \mathscr{X}_{m_{0}}$, найдется множество $P \in \mathscr{B}$, для которого вьполнены условия (i) и (ii) для $m=m_{0}$ и справедливо равенство $X_{m_{0}}=\operatorname{pr} P$. Положим $P_{0}=P$.

Допустим, что $m_{n}$ и $P_{n} \in \mathscr{B}_{m_{n}}$ уже определены. Тогда $P_{n}=\mathrm{B}^{(m)}$ для подходящего $m>m_{n}$. Напомним, что $X_{m} \in \mathscr{X}_{m}$; следовательно, имеется множество $B \in \mathscr{B}$, удовлетворяюшее (i) и (iі) для этого $m$, и $X_{m}=\operatorname{pr} B$. Заметим, что случай $X_{m} \cap \operatorname{pr~B}^{(m)}$ в (ii) здесь невозможен. В самом деле, $\operatorname{pr~B}^{(m)}=\operatorname{pr} P_{n}=X_{m_{n}}$; однако $X_{m} \cap X_{m_{n}}$ непусто по выбору множеств $X_{m}$. Значит, $B \subseteq \mathrm{B}^{(m)}=P_{n}$. Остается положить $m_{n+1}=m$ и $P_{n+1}=B$.

Наконец, требование “генеричности" (4) гарантировано из (ii).

ЗАмечАниЕ 11. Нетрудно проверить, что польская сеть $\left\{\mathscr{X}_{m}: m \in \omega\right\}$, даваемая леммой, удовлетворяет следующему условию монотонности: если $Y$ есть $\Sigma_{1}^{1}$-множество и $\varnothing \neq Y \subseteq X \in \mathscr{X}_{m}$, то $Y \in \mathscr{X}_{m}$.

2.3. Некоторые следствия. Мы рассмотрим приложения леммы 9 , в том числе для некоторых производных топологий.

ОПРЕДЕЛЕНИЕ 12 . Пусть $n \geqslant 1$. $\mathscr{T}_{n}$ будет обозначать топологию $\mathscr{T}$ пространства $\mathscr{N}^{n}$. (Заметим, что $\left\{\mathscr{N}^{n} ; \mathscr{T}_{n}\right\}$ гомеоморфно $\langle\mathscr{N} ; \mathscr{T}\rangle$.)

$\mathscr{T}^{n}$ есть тихоновское произведение $n$ копий $\mathscr{T}=\mathscr{T}_{1}$.

$\mathscr{T}_{n+m}$ есть произведение $\mathscr{T}_{n} \times \mathscr{T}_{m}$, топология на $\mathscr{N}^{n+m}$.

СЛЕДСТВИЕ 13. Все топологии $\mathscr{T}_{n}$ и $\mathscr{T}^{n}$ являются бэровскими. ${ }^{13}$

ДокАЗАТЕльСтво. Во-первых, произведение двух топологий, обладающих польской сетью, само имеет такую сеть (она состоит из декартовых произведений множеств, входящих в исходные сети). Затем, чтобы доказать, что топология с польской сетью является бэровской, используем простейшее доказательство бэровости полных метрических пространств.

Топология $\mathscr{T}_{n}$ содержит $\mathscr{T}^{n}$ и, на самом деле, строго сильнее, чем $\mathscr{T}^{n} ;$ например, диагональ $\Delta(\mathscr{N})=\{\langle x, x\rangle: x \in \mathscr{N}\}$ открыта в $\mathscr{T}_{2}$, но не в $\mathscr{T}^{2}$. Однако произведение топологий достаточно плотно в топологии произведения.

ЛЕмма 14. Пусть множсество $V \subseteq \mathscr{N}$ T-открыто, а $D \subseteq V$ имеет вторую категорию в $V$ в смысле $\mathscr{T}$. Тогда $D \times D$ плотно в $V \times V$ в смисле $\mathscr{T}_{2}$.

ДокаЗАТЕЛЬСтво. Считаем для простоты, что $V=\mathscr{N}$. Пусть $\bigcap_{n} D_{n} \subseteq D$, где все множества $D_{n} \subseteq \mathscr{N}$ являются $\mathscr{T}$-открытыми и плотными в $\mathscr{N}$. Тогда каждое множество $D_{n}^{\prime}=D_{n} \times \mathscr{N}$ открыто и плотно в $\mathscr{T}_{2}$. (Действительно, проекция $\operatorname{pr} A$

\footnotetext{
${ }^{13}$ Бәровские топологии характеризуются свойством: все множества второй категории плотны. Множество второй категории - это множество, включающее счетное пересечение плотных открытых множеств. (Прямое доказательство бэровости $\mathscr{T}$ приведено в [20].)
} 
любого $\Sigma_{1}^{1}$-множества $A$ есть $\Sigma_{1}^{1}$-множество в $\mathscr{N}$.) Аналогично, каждое множество $D_{n}^{\prime \prime}=\mathscr{N} \times D_{n}$ открыто и плотно. В то же время мы имеем $\bigcap_{n}\left(D_{n}^{\prime} \cap D_{n}^{\prime \prime}\right) \subseteq D \times D$. Остается применить следствие 13.

Следующая лемма будет использована ниже при исследовании борелевских отношений эквивалентности.

Пусть R является отношением эквивалентности на $\mathscr{N}$. Положим

$$
\mathrm{R}^{(n)}=\left\{\left\langle x_{1}, \ldots, x_{n}\right\rangle: \forall i\left(x_{i} \mathrm{R} x_{i+1}\right)\right\} .
$$

Для любых $n$ и $m,\left\langle\mathrm{R}^{(n+m)} ; \mathscr{T}_{n+m}\right\rangle$ будет обозначать множество $\mathrm{R}^{(n+m)} \subseteq \mathscr{N}^{n+m}$ с топологией, унаследованной из пространства $\left\langle\mathscr{N}^{n+m} ; \mathscr{T}_{n+m}\right\rangle$.

ЛЕмма 15. Пусть $\mathrm{R}$ есть $\Sigma_{1}^{1}$-отношение әквивалентности на $\mathscr{N}, n^{\prime} \leqslant n u$ $m^{\prime} \leqslant m$. Тогда проектирование $\pi: \mathscr{N}^{n} \times \mathscr{N}^{m}$ на $\mathscr{N}^{n^{\prime}} \times \mathscr{N}^{m^{\prime}}$ есть открытое непреривное отображсение $\left\langle\mathrm{R}^{(n+m)} ; \mathscr{T}_{n+m}\right\rangle$ на $\left\langle\mathrm{R}^{\left(n^{\prime}+m^{\prime}\right)} ; \mathscr{T}_{n^{\prime}+m^{\prime}}\right\rangle$.

ДокАЗАТЕЛЬСТво. Пусть, для простоты, $m=n=2, m^{\prime}=n^{\prime}=1$; таким образом, $\pi\left(x_{1}, x_{2} ; y_{1}, y_{2}\right)=\left\langle x_{1}, y_{1}\right\rangle$. Опуская простую проверку непрерьвности, сосредоточимся на доказательстве открытости, где, в частности, будет важно особое строение множества $\mathrm{R}^{(n+m)}$. Рассмотрим пару $\mathscr{T}_{2}$-базовых, т.е. класса $\Sigma_{1}^{1}$, множеств $U, V \subseteq \mathscr{N}^{2}$. Докажем, что проекция $O=\pi\left((U \times V) \cap \mathrm{R}^{(4)}\right) \mathscr{T}^{2}$-открыта в $\mathrm{R}^{(2)}=\mathrm{R}$. Используя свойства замкнутости эффективных классов (см. раздел 1.5), мы находим, что множества

$$
U^{\prime}=\left\{x_{1}: \exists x_{2}\left[U\left(x_{1}, x_{2}\right) \& x_{1} \mathrm{R} x_{2}\right]\right\} \quad \text { и } V^{\prime}=\left\{y_{1}: \exists y_{2}\left[V\left(y_{1}, y_{2}\right) \& y_{1} \mathrm{R} y_{2}\right]\right\}
$$

принадлежат $\Sigma_{1}^{1}$, т.е. $\mathscr{T}$-открыты. Однако $O=\left(U^{\prime} \times V^{\prime}\right) \cap \mathrm{R}$.

2.4. Пространства Шоке. Теперь мы представим более традиционный аппарат исследования топологий $\mathscr{T}$, основанный на играх Шоке.

Игра ШІоке $C_{\mathscr{X}}$ в топологическом пространстве $\mathscr{X}$ происходит следующим образом. Участвуют два игрока, $\beta$ и $\alpha$, причем начинает $\beta$. Ходами в игре являются непустые открытые подмножества $\mathscr{X}$, причем каждый ход должен быть подмножеством предшествуюшего хода противника. Получается последовательность ходов

$$
U_{0} \supseteq V_{0} \supseteq U_{1} \supseteq V_{1} \supseteq \cdots \supseteq U_{n} \supseteq V_{n} \supseteq \cdots \quad\left(U_{n} \text { и } V_{n} \text { открыты }\right),
$$

причем ходы $U_{n}$ делаются игроком $\beta$, а ходы $V_{n}-$ игроком $\alpha$. Каждый из игроков перед своим очередным ходом знает все, что происходило в игре до этого хода.

Наконец, в результате игры побеждает $\alpha$, когда $\bigcap_{n} U_{n}\left(=\bigcap_{n} V_{n}\right) \neq \varnothing$.

$\mathscr{X}$ есть пространство Шоке или пространство со свойством Шоке, если $\alpha$ имеет выигрывающую стратегию. ${ }^{14}$

\footnotetext{
${ }^{14}$ То есть правило, которое предписьвает $\alpha$, как ему играть в зависимости от игры $\beta$, чтобы выиграть игру, как бы ни играл игрок $\beta$. Это понятие технически реализуется в виде функции $\tau$, определенной на кортежах $\left\langle U_{0}, \ldots, U_{n}\right\rangle$ открытых множеств и принимающей значения также среди открытых множеств, так что $V_{n}=\tau\left(U_{0}, \ldots, U_{n}\right) \subseteq U_{n}$. См. [20] о бесконечных играх с полной информацией, к которым относится и игра $C \mathscr{X}$.
} 
Модифицированная, сильная игра Шоке $C_{\mathscr{X}}^{\prime}$ дает некоторьй дополнительньй шанс игроку $\beta$. Именно, вместе с каждым ходом $U_{n}, \beta$ делает дополнительный ход $x_{n} \in U_{n}$, обязывающий $\alpha$ ответить ходом $V_{n}$ так, чтобы $x_{n} \in V_{n}$ (и, по-прежнему, $V_{n} \subseteq U_{n}$ ).

Результат определяется, как в игре $C_{\mathscr{X}}: \alpha$ вьпграл, если $\bigcap_{n} V_{n} \neq \varnothing$.

Наконец, $\mathscr{X}$ назьвается пространством с сильным свойством Шоке, если $\alpha$ имеет вьпгрьвающую стратегию в игре $C_{\mathscr{X}}^{\prime}$.

ЛЕмма 16. Полные метрические пространства и пространства, обладающие польской сетью, имеют сильное свойство Шоке.

ДокАЗАТЕльство. В случае полного метрического пространства, $\alpha$ может играть так, чтобы замыкание $V_{n}$ было подмножеством $U_{n}$ и имело диаметр $<n^{-1}$. Если имеется польская сеть $\left\{\mathscr{X}_{m}: m \in \omega\right\}$, то игроку $\alpha$ достаточно делать каждый ход $V_{n}$ так, чтобы $V_{n} \in \mathscr{X}_{n}$.

В свою очередь, и сильное свойство Шоке влечет за собой определенную “регулярность”, позволяя получать некоторые обычные следствия полноты.

ПРЕДЛОЖЕНИЕ 17 (Харрингтон, Кекрис и Луво [12]).

1. Сильное свойство Шоке влечет за собой свойство Шоке.

2. Пространство Шоке есть бәровское пространство.

3. Если $X, Y$ - пространства Шоке, то $X \times Y$ - также пространство ШІоке. То же для пространств с сильным свойством Шоке.

4. Если $\mathscr{X}$ имеет сильное свойство Шоке, то любое непустое $\mathbb{G}_{\delta}$-множество $X \subseteq \mathscr{X}$ имеет сильное свойство Шоке в унаследованной топологии.

ДокаЗАТЕльство. 1. Очевидно: $C_{\mathscr{X}}$-стратегия игрока $\alpha$ есть его $C_{\mathscr{X}}^{\prime}$-стратегия, игнорируюшая ходы $x_{n}$.

2. Пусть каждое $D_{n} \subseteq \mathscr{X}$ открыто и плотно, а $U$ открыто и непусто; докажем, что $I=U \cap \bigcap_{n} D_{n}$ также непусто. Заставим $\beta$ играть в $C \mathscr{X}$, делая ходы $U_{n} \subseteq D_{n}$ (используется открытость и плотность $D_{n}$ ), а начальный ход $U_{0} \subseteq D_{0} \cap U$. Вьпгрывающая стратегия $\alpha$ доказьвает непустоту $I$.

3. Упражнение: $\alpha$ "расщепляет" игру $C \mathscr{X} \times \mathscr{Y}$ на игры $C \mathscr{X}$ и $C \mathscr{Y}$, предварительно сужая каждый ход $U_{n}$ противника до произведения $U_{n}^{x} \times U_{n}^{y} \subseteq U_{n}$, где $U_{n}^{x}$ и $U_{n}^{y}$ открыты в $\mathscr{X}$ и $\mathscr{Y}$, соответственно.

4. Пусть $X=\bigcap_{n} G_{n}$, где каждое $G_{n}$ открыто в $\mathscr{X}$. Чтобы выпграть $C_{X}^{\prime}$, игрок $\alpha$ действует следуюшим образом. Пусть $\left\langle U_{0}, x_{0}\right\rangle, \ldots,\left\langle U_{n}, x_{n}\right\rangle$ - начальные ходы $\beta$ в игре $C_{X}^{\prime}$. Тогда $x_{i} \in U_{i}=X \cap \mathscr{U}_{i}$, где множества $\mathscr{U}_{i} \subseteq \mathscr{X}$ открыты для всех $i \leqslant n$. Допустим, что $C_{\mathscr{X}}^{\prime}$-стратегия рекомендует для $\alpha$ ход $\mathscr{V}_{n}$ в ответ на ходы $\left\langle\mathscr{U}_{0}, x_{0}\right\rangle,\left\langle\mathscr{U}_{1}, x_{1}\right\rangle, \ldots,\left\langle\mathscr{U}_{n}, x_{n}\right\rangle$ игрока $\beta$. Найдется открытое $\mathscr{V}^{\prime} \subseteq \mathscr{X}$ такое, что $x_{n} \in \mathscr{V}^{\prime} \subseteq \mathscr{V}_{n} \cap G_{n}$. Теперь $\alpha$ делает ход $V_{n}=\mathscr{V}^{\prime} \cap X$ в игре $C_{X}^{\prime}$.

Мы закончим этот раздел изложением прямого доказательства свойства Шоке для топологий $\mathscr{T}$. Читатель может найти любопытные параллели этого рассуждения с доказательством леммы 9. 
Лемма 18. Пусть $p \in \mathscr{N}$ u $n \geqslant 1$. Тогда топология $\mathscr{T}$ на $\mathscr{N}^{n}$ имеет сильное свойство Ноке.

ДокАЗАТЕльСтво. Каждое $\mathscr{N}^{n}$ с топологией $\mathscr{T}$ гомеоморфно $\langle\mathscr{N} ; \mathscr{T}\rangle$, поэтому можно считать, что $n=1$. Напомним, что базу $\mathscr{T}$ образуют $\Sigma_{1}^{1}$-множества, а каждое такое множество $X \subseteq \mathscr{N}$ есть проекция некоторого $\Pi_{1}^{0}$-множества $F \subseteq \mathscr{N}^{2}$. Это намечает главную идею доказательства: использовать полноту пространств $\mathscr{N}^{m}$ в польской метрике (см. раздел 1.1).

В обозначениях из доказательства леммы 9, искомая стратегия для $\alpha$ в игре $C^{\prime}=C_{\langle\mathscr{N} ; \mathscr{T}\rangle}^{\prime}$ может быть описана следующим образом.

Пусть $\left\langle U_{0}, x_{0}\right\rangle,\left\langle U_{1}, x_{1}\right\rangle,\left\langle U_{2}, x_{2}\right\rangle, \ldots$ - последовательность ходов $\beta$. Вместе со своими ходами $V_{0}, V_{1}, V_{2}, \ldots$, которые в данном случае будут множествами класса $\Sigma_{1}^{1}$, а не просто $\mathscr{T}$-открытыми, причем $x_{n} \in V_{n}$, игрок $\alpha$ строит последовательность $\Pi_{1}^{0}$-множеств $F_{n} \subseteq \mathcal{N} \times \mathscr{N}^{n}$ так, что

(2) $\quad x_{n} \in V_{n}=\operatorname{pr} F_{n} \subseteq U_{n}, \quad \operatorname{pr}_{<n} F_{n} \subseteq F_{n-1}, \quad \operatorname{diam}_{p_{m}} F_{n} \leqslant n^{-1}$ при $m \leqslant n$.

Благодаря полноте, условие на диаметры влечет существование единственной последовательности точек $\vec{x}=\left\langle x, x_{0}, x_{1}, x_{2}, x_{3}, \ldots\right\rangle \in \mathscr{N}^{1+\omega}$, удовлетворяющей $\vec{x}\left\lceil\leqslant n \in F_{n}\right.$ для всех $n$. Тогда $x \in \bigcap_{n} V_{n}$, что и требуется.

Остается проверить, что $\alpha$ может обеспечить (2) для всех $n$ правильной игрой. Итак, пусть $\beta$ сделал очередной ход $\left\langle U_{n}, x_{n}\right\rangle$; при этом выполнено $x_{n} \in U_{n} \subseteq V_{n-1}=$ pr $F_{n-1}$. Поскольку $U_{n}$ открыто в топологии $\mathscr{T}$, найдется непустое $\Sigma_{1}^{1}$-множество $U_{n}^{\prime} \subseteq U_{n}$ и найдется $\Pi_{1}^{0}$-множество $P \subseteq \mathscr{N} \times \mathscr{N}$ такое, что $U_{n}^{\prime}=\operatorname{pr} P$. Тогда множество $F=\left\{\langle x, \boldsymbol{x}, y\rangle:\langle x, \boldsymbol{x}\rangle \in F_{n-1} \& P(x, y)\right\}$ принадлежит $\Pi_{1}^{0}$ и удовлетворяет $x_{n} \in \operatorname{pr} F=U_{n}^{\prime}$ и $\operatorname{pr}_{<n} F \subseteq F_{n-1}$. (Заметим, что $F \subseteq \mathscr{N} \times \mathscr{N}^{n}$.) Разобьем $F$ на счетное число $\Pi_{1}^{0}$-множеств с проекциями диаметра $<n^{-1}$ посредством пересечения с "малыми” польскими базовыми окрестностями в $\mathcal{N} \times \mathscr{N}^{n}$. Одно из этих множеств, скажем, $F^{\prime} \subseteq F$, удовлетворяет $x_{n} \in \operatorname{pr} F^{\prime}$. Полагаем $F_{n}=F^{\prime}$ и $V_{n}=\operatorname{pr} F^{\prime}$ (ответ $\alpha$ ).

\section{3. Первое приложение: ко-суслинские отношения эквивалентности}

Некоторое обсуждение необходимо перед технической частью этого раздела, чтобы правильно представить смысл результатов.

Напомним, что континуум-гипотеза $(\mathrm{CH})$ Кантора - это утверждение о том, что нет ни одной мошности строго между счетной мощностью $\aleph_{0}$ и мощностью континуума $\mathfrak{c}=2^{\aleph_{0}}$ (которая строго больше $\aleph_{0}$ ). После работ К. Гёделя 30-х годов и П. Коэна начала 60-х определенно установлено, что современная математика (по крайней мере, настолько, насколько она базируется на теории множеств Цермело-Френкеля ZFC) не позволяет дать ответ "да" или "нет" на вопрос: верна ли СН?

Однако весьма плодотворным оказался подход, направленный на проверку СН в определенных классах множеств. В частности, Александров [9] и Хаусдорф [13] доказали, что СН верна в классе борелевских (в смысле польской топологии) множеств вещественной прямой $\mathbb{R}$ или, что в данном случае равносильно, пространства $\mathscr{N}$. Иными словами, борелевское $X \subseteq \mathscr{N}$ не может иметь промежуточной мощности. Нет 
контрпримеров и в более широком классе $\boldsymbol{\Sigma}_{1}^{1}$-множеств - это теорема Суслина [3], утверждающая, что, более того, всякое несчетное $\boldsymbol{\Sigma}_{1}^{1}$-множество имеет совершенное ${ }^{15}$ подмножество. Однако уже в классе $\Pi_{1}^{1}$ контрпримеры могут быть. (См. работу автора [4], где дан более подробный анализ этого круга вопросов.)

Есть однако и другой подход к изучению СН в определенных классах, состоящий в том, что мы ищем контрпримеры не в виде точечных множеств, а в виде фактор-множеств, т.е. исследуется вопрос: сколько классов эквивалентности может иметь отношение эквивалентности того или иного типа. Картина здесь получается отчасти похожая на ту, что мы видим для множеств, однако с обращением, т.е. число классов эквивалентности $\boldsymbol{\Pi}_{1}^{1}$-отношения не может быть промежуточной мощностью, в то время как число классов эквивалентности $\boldsymbol{\Sigma}_{1}^{1}$-отношения-может (см. конец этого раздела).

Вторая часть этого утверждения (о $\boldsymbol{\Sigma}_{1}^{1}$-отношениях $)$ следует из сказанного вьше о $\boldsymbol{\Pi}_{1}^{1}$-множествах; первая же часть весьма сложна; фактически до сих пор не известно ни одного ее доказательства, которое было бы проведено в рамках классических методов дескриптивной теории множеств.

ТЕОрема 19 (Сильвер [23]). Пусть Е является П 1 -отношением әквивалентности на $\mathcal{N}$. Тогда либо $\mathrm{E}$ имеет конечное или счетное число классов, либо существует совершенное множсество попарно Е-неэквивалентньх точек.

Заметим, что результат автоматически переносится на все полные сепарабельные метрические пространства, так как каждое такое пространство допускает борелевский (в частности, сохраняющий $\Pi_{1}^{1}$ ) изоморфизм на $\mathcal{N}$.

ДокАЗАтЕльство. 16 Допустим, что Е имеет несчетно много классов эквивалентности, и докажем, что тогда существует совершенное множество попарно неэквивалентных точек. Доказательство этого положения содержит идею и две технических части.

Идея. Допустим, что нам удалось найти непустое открытое в польской топологии $\mathscr{N}$ множество $H \subseteq \mathscr{N}$ такое, что Е имеет первую категорию на $H^{2}=H \times H$; другими словами, $\mathrm{E} \cap H^{2} \subseteq \bigcup_{n} P_{n}$, где все $P_{n} \subseteq H^{2}$ нигде не плотны. В этом случае мы легко строим систему $\left\langle X_{s}: s \in 2^{<\omega}\right\rangle$ непустых открыто-замкнутых множеств $X_{s} \subseteq H$ такую, что

(a) $X_{s^{\wedge} i} \subseteq X_{s}, X_{s^{\wedge}} \cap \cap X_{s^{\wedge}}=\varnothing$,

(b) $\left(X_{s} \times X_{t}\right) \cap \bigcup_{m \leqslant n} P_{m}=\varnothing$ при $s, t \in 2^{n}, s \neq t$,

(c) $\operatorname{diam} X_{s} \leqslant m^{-1}$ при $s \in 2^{m}$,

где $2^{n}$ есть совокупность всех последовательностей 0 и 1 длины $n, 2^{<\omega}=\bigcup_{n \in \omega} 2^{n}, s^{\wedge} i$ имеет очевидный смысл, a $\operatorname{diam} X$ обозначает диаметр $X$ в польской метрике. Тогда

\footnotetext{
${ }^{15}$ То есть непустое, замкнутое и не имеющее изолированных точек в смысле польской топологии. Такие множества имеют мощность континуума.

${ }^{16}$ Предлагаемое доказательство принадлежит Харрингтону и взято из статьи Мартина и Кекриса [20], с некоторым изменением, состоящим в том, что вместо игр Шоке использована техника польских сетей. Более сложное доказательство самого Сильвера [23] основано на технике форсинга; похожее доказательство приведено Миллером [21].
} 
множество $X=\bigcap_{n} \bigcup_{s \in 2^{n}} X_{s}$ есть искомое совершенное множество из попарно неэквивалентных точек.

K сожалению, мы не можем утверждать, что множество $H$ указанного вида действительно существует - в польской топологии. Это тот момент, когда топологии $\mathscr{T}(p)$ входят в игру.

Техническая часть 1. Во-первых, поскольку $\mathrm{E} \in \Pi_{1}^{1}$, найдется $p \in \mathscr{N}$ такое, что $\mathrm{E}$ является $\Pi_{1}^{1}(p)$-отношением. Будем считать, что $\mathrm{E}$ принадлежит $\Pi_{1}^{1}$; если это фактически не так, то просто параметр $p$ равномерно присутствует в выкладках, т.е., скажем, $\mathscr{T}$ меняется на $\mathscr{T}(p)$ и т.п. (см. замечание в конце раздела 2.1).

Мы укажем непустое $\mathscr{T}$-открытое множество $H$ такое, что Е имеет первую категорию на $H^{2}=H \times H$ в смысле $\mathscr{T}^{2}$.

В сушности, идея весьма проста: удалим те классы Е-эквивалентности, которые открыты в топологии $\mathscr{T}$. В польской топологии этот номер не пройдет: нет никакой гарантии, что после удаления открытых классов останется открытое множество. В топологии Ганди-Харрингтона, как мы увидим, все будет в порядке, благодаря ее дескриптивньм свойствам.

Мно жество Харрингтона $H$ определяется равенством

$$
H=\left\{x \in \mathscr{N}: \text { нет такого } \Delta_{1}^{1} \text {-множества } B \text {, что } x \in B \subseteq[x]\right\},
$$

где $[x]=[x]_{\mathrm{E}}=\{y: x \mathrm{E} y\}$ есть класс Е-эквивалентности $x$.

Заметим, что $H$ непусто; действительно, иначе каждый Е класс был бы объединением $\Delta_{1}^{1}$-множеств, а поскольку имеется лишь счетное число последних, в этом случае Е имело бы не более чем счетное число классов - противоречие с предположением в начале доказательства.

ЛЕмма 20. Н открыто в $\mathscr{T} ;$ более того, $H$ принадлежит $\Sigma_{1}^{1}$.

ДокаЗАТЕльСтво. В самом деле,

$$
x \in H \longleftrightarrow \forall B \in \Delta_{1}^{1}(x \in B \rightarrow \exists y \in B x \text { E } y)
$$

Пусть $p=\mathbf{0}=\omega \times\{0\}$ (тождественньй ноль, т.е., скажем, $\Delta_{1}^{1}=\Delta_{1}^{1}(\mathbf{0})$ ). Используя множества $\mathbf{W}$ и $\mathbf{D}_{n}$, даваемые принципом 4 (часть 1 ) раздела 1 , мы приводим правую часть (3) к виду

$$
\forall n\left[\mathbf{W}(\mathbf{0}, n) \& x \in \mathbf{D}_{n}(\mathbf{0}) \rightarrow \exists y\left(y \in \mathbf{D}_{n}(\mathbf{0}) \& x \in \mathbb{E} y\right)\right]
$$

Это выражение без труда можно преобразовать к $\Sigma_{1}^{1}$, используя свойства множеств $\mathbf{W}$ и $\mathbf{D}_{n}$.

ЛЕмма 21. Е имеет первую категорию на $\mathrm{H}^{2}$ в смысле $\mathscr{T}^{2}$. 
ДокАЗАТЕльСтво. Мы напомним, что $\mathbf{E}$ есть $\Pi_{1}^{1}$, т.е. ко-суслинское множество в польской топологии, следовательно, и в $\mathscr{T}$. Отсюда следует, что Е имеет свойство Бэра в смысле $\mathscr{T}$, так как известно, что свойство Бэра сохраняется при действии $A$-операции. Таким образом, по теореме Улама-Куратовского достаточно доказать, что $H_{x}=H \cap[x]=\{y \in H: x \mathrm{E} y\}$ имеет первую категорию в смысле $\mathscr{T}$, каково бы ни было $x \in H$.

Как и вьше, $H_{x}$ имеет свойство Бэра в смысле $\mathscr{T}$. Значит, чтобы проверить, что $H_{x}$ имеет первую $\mathscr{T}$-категорию, достаточно установить, что $H_{x}$ не может иметь второй категории ни на каком непустом $\Sigma_{1}^{1}$-множестве $D \subseteq H$.

Пусть, напротив, $H_{x}$ имеет вторую категорию в смысле $\mathscr{T}$ на непустом $\Sigma_{1}^{1}$-множестве $U \subseteq H$. Множество $D^{\prime}=\left(H_{x} \cap U\right) \times\left(H_{x} \cap U\right)$ плотно в $U^{2}=U \times U$ в смысле $\mathscr{T}_{2}$ по лемме 14 . Таким образом, $D^{\prime}$ непусто пересекает любое непустое $\Sigma_{1}^{1}$-множество $P \subseteq U^{2}$. В частности, если множество $P=\left\{\langle y, z\rangle \in U^{2}: y \not E z\right\}$ непусто, то $P \cap D^{\prime} \neq \varnothing$.

Допустим, что $\langle y, z\rangle \in P \cap D^{\prime}$. Тогда как $y$, так и $z$ принадлежат $H_{x}$, т.е. $y \mathrm{E} z$, что противоречит предположению $\langle y, z\rangle \in P$. Следовательно, на самом деле $P$ - пустое множество, откуда вытекает, что $U \subseteq[x]$.

Но тогда сам класс эквивалентности $[x]$ принадлежит $\Pi_{1}^{1}$, поскольку $y \mathrm{E} x$ равносильно $\forall z[z \in U \rightarrow z \mathrm{E} y]$. Итак, $\Sigma_{1}^{1}$-множество $U$ включено в $\Pi_{1}^{1}$-множество $[x]$. По теореме отделимости (следствие 3 ), найдется $\Delta_{1}^{1}$-множество $B$ такое, что $U \subseteq$ $B \subseteq[x]$. Возьмем произвольное $x^{\prime} \in U$. Тогда $x^{\prime} \mathrm{E} x$, т.е. мы имеем $x^{\prime} \in B \subseteq\left[x^{\prime}\right]$, откуда следует, что $x^{\prime} \notin H$. Однако $x^{\prime} \in U \subseteq H$, противоречие.

Техническая часть 2. Модифицировав рассуждение, приведенное в начале доказательства теоремы, мы построим совершенное множество $X$ попарно Е-неэквивалентных точек. Модификация состоит в том, что польская сеть топологии $\mathscr{T}$ заменит полноту польской топологии $\mathcal{N}$.

Пусть, по доказанному, $\mathrm{E} \cap H^{2} \subseteq \bigcup_{n} P_{n}$, где каждое $P_{n} \subseteq \mathscr{N}^{2}$ нигде не плотно в $\mathscr{T}^{2}$. Пусть $\left\{\mathscr{X}_{n}: n \in \omega\right\}$ - польская сеть, даваемая леммой 9 для топологии $\mathscr{T}$.

Не составит большого труда построить семейство $\Sigma_{1}^{1}$-множеств $X_{s} \subseteq H\left(s \in 2^{<\omega}\right)$, удовлетворяюшее условиям (а) и (b) (см. вьше) и следуюшему требованию вместо (c):

( $\left.{ }^{\prime}\right)$ если $s \in 2^{m}$, то $X_{s} \subseteq X_{s}^{\prime}$ для некоторого $X_{s}^{\prime} \in \mathscr{X}_{m}$.

При любом $a \in 2^{\omega}$ последовательность множеств $X_{\mathbf{a} \uparrow m}, m \in \omega$, содержит ровно одну точку $x_{a}$ в пересечении, так как $X_{a \uparrow m} \in \mathscr{X}_{m}$. По построению, если $a \neq a^{\prime}$, то $\left\langle x_{a}, x_{a^{\prime}}\right\rangle \notin P_{m}$ для всех $m$, так что $x_{a} E x_{a^{\prime}}$ и, в частности, $x_{a} \neq x_{a^{\prime}}$. Следовательно, множество $X=\left\{x_{a}: a \in 2^{\omega}\right\}$ есть совершенное (даже гомеоморфное канторову дисконтинууму) множество попарно Е-неэквивалентных точек.

ЗАмЕчАниЕ 22. Дадим краткое описание конструкции множества попарно неэквивалентных точек в статье [12], где для обеспечения непустоты пересечений вдоль каждого пути $a \in 2^{\omega}$ используется игра Шоке.

Мы строим два индексированных семейства $\left\{U_{s}: s \in 2^{<\omega}\right\}$ и $\left\{V_{s}: s \in 2^{<\omega}\right\}$ непустых $\Sigma_{1}^{1}$-подмножеств $\mathscr{N}$ так, чтобы, во-первых, семейство множеств $U_{s}$ удовле- 
творяло условиям (a), (b), (c), а, во-вторых, при любом $a \in 2^{\omega}$ последовательность множеств

$$
U_{a \uparrow 0}=U_{\Lambda}, V_{a \uparrow 0}=V_{\Lambda}, U_{a \uparrow 1}, V_{a \uparrow 1}, U_{a \uparrow 2}, V_{a \uparrow 2}, \ldots, U_{a \uparrow n}, V_{a \uparrow n}, \ldots
$$

отвечала вьигрывающей стратегии игрока $\alpha$ в игре Шоке $C_{\langle\mathcal{N} ; \mathscr{T}\rangle}$ (которую $\alpha$ имеет благодаря лемме 18). ${ }^{17}$ Тогда пересечение $X_{a}=\bigcap_{n} V_{a \nmid n}$ непусто, каково бы ни было $a \in 2^{\omega}$; на самом деле, благодаря условию (c), наложенному на диаметры, $X_{a}$ содержит ровно одну точку $x_{a}$, и т. д.

Отношения эквивалентности класса $\Sigma_{1}^{1}$. Это направление сейчас вызьвает, пожалуй, наибольший интерес, предлагая весьма интересные и трудные вопросы, в частности, в связи с возможностью получать аналоги теоремы Сильвера.

К сожалению, сама теорема 19 не распространяется на $\boldsymbol{\Sigma}_{1}^{1}$-отношения. Рассмотрим неборелевское $\Pi_{1}^{1}$-множество $C \subseteq \mathscr{N}$, разбитое на борелевские конституанты как $C=$ $\bigcup_{\alpha<\omega_{1}} C_{\alpha}$. Определим отношение Е посредством

$$
x \mathrm{E} y, \text { если и только если } \exists \alpha\left(x \in C_{\alpha} \& y \in C_{\alpha}\right) \vee(x \notin C \& y \notin C) \text {. }
$$

Это $\boldsymbol{\Sigma}_{1}^{1}$-отношение, не допускающее совершенных множеств попарно неэквивалентных точек согласно принципу ограничения классической дескриптивной теории, но имеющее $\aleph_{1}$ (непустых) классов эквивалентности.

Бэрджес [10] установил, что данньй пример достаточно представителен: вообще, отношение класса $\boldsymbol{\Sigma}_{1}^{1}$, для которого нет совершенного множества попарно неэквивалентных точек - такие отношения называются тонкими - имеет $\leqslant \aleph_{1}$ классов эквивалентности. До сих пор остается открытьм вопрос, возможен ли абсолютный список классов.

Представляется желательным (и естественньм) следуюшее. Рассмотрим транзитивный класс $M \subseteq \mathrm{V}$ в теоретико-множественном универсуме $\mathrm{V}$, которьй сам является моделью ZFC, например, класс L всех конструктивных множеств. Будем предполагать также, что $M$ правильно вычисляет $\aleph_{1}$, т.е. $\aleph_{1}=\aleph_{1}^{M}$. (Иначе постановка вопроса теряет смысл.) Пусть, наконец, Е есть тонкое $\Sigma_{1}^{1}$-отношение эквивалентности. Верно ли, что тогда любой (непустой) класс Е имеет представителя в $M$ ? Положительный ответ получен Хъёртом [14] в двух случаях:

1) V есть генерическое расширение $M$,

2) некоторый дериват гипотезы существования измеримого кардинала; однако обший случай остается открытым.

Исследование тонких $\boldsymbol{\Sigma}_{1}^{1}$-отношений сушественно облегчается в случае, когда мы дополнительно требуем, чтобы все классы эквивалентности были борелевскими множествами ограниченного ранга - т.е. все они принадлежали некоторому одному борелевскому классу $\Sigma_{\alpha}^{0}\left(\alpha<\omega_{1}\right)$; такие отношения называются лузинскими, поскольку

\footnotetext{
${ }^{17}$ Формально это означает, что $V_{a \uparrow n}=\tau\left(U_{a \uparrow 0}, U_{a \uparrow 1}, \ldots, U_{a \uparrow n}\right)$ при любом $n$, где $\tau$ - выигрьвающая стратегия $\alpha$.
} 
Н.Н. Лузин был инициатором их изучения. ${ }^{18}$ Хотя и здесь нельзя утверждать, что каждое лузинское $\boldsymbol{\Sigma}_{1}^{1}$-отношение имеет лишш счетно много классов (соответствуюшие контрпримеры были указаны Сами [22]), однако в этом случае классы допускают вполне абсолютную нумерацию счетными ординалами. Более того, в предположении, что $\mathscr{N} \cap \mathrm{L}[x]$ счетно для любого $x \in \mathscr{N}$, любое лузинское даже $\boldsymbol{\Delta}_{2}^{1}$-отношение имеет лишш счетно много классов, см. [26] и [3] (специально для эквивалентностей, порождаемых разбиением на конституанты).

\section{4. Классификация борелевских отношений эквивалентности}

Мы продолжаем изложение результатов по отношениям эквивалентности, которые на самом деле допускают значительно более обстоятельный анализ, чем альтернатива "счетно много классов или совершенное множество попарно неэквивалентных точек", представленная в разделе 3. Этот анализ связан с вопросами сравнения и классификикции отношений эквивалентности.

4.1. Сглаженные отношения. Этот тип отношений эквивалентности приобрел важное значение, в частности, в связи с некоторыми задачами теории групा борелевских преобразований и теории меры.

ОПРЕДЕЛЕНИЕ 23. Отношение эквивалентности Е на пространстве $\mathscr{X}$ называется сглаженны. ${ }^{19}$, когда сушествует борелевская функция $f: \mathscr{X} \rightarrow \mathscr{N}$ такая, что $x \mathrm{E} y \longleftrightarrow f(x)=f(y)$.

Другими словами, сглаженные отношения обладают борелевскими инвариантами. $\mathscr{N}$ может быть заменено любым совершенным польским пространством $\mathscr{Y}$, поскольку все эти пространства борелевски изоморфны $\mathcal{N}$.

Часто бьвает полезньм следуюший критерий: $\mathrm{E}$ - сглаженное отношение, если и только если сушествует семейство борелевских множеств $A_{n}$ такое, что $x \mathrm{E} y \longleftrightarrow$ $\forall n\left(x \in A_{n} \longleftrightarrow y \in A_{n}\right)$ (разделяющее семейство).

$\mathrm{K}$ этой категории относятся, например, некоторые отношения между матрицами, возникающие из приведения к какой-либо канонической форме.

Примером не сглаженного отношения может служить отношение Витали $\mathrm{E}_{\mathrm{V}}$ на вешественной прямой $\mathbb{R}$. Чтобы увидеть, что $\mathrm{E}_{\mathrm{V}}$ - несглаженное отношение, допустим, что, напротив, борелевская функция $f: \mathbb{R} \rightarrow \mathscr{N}$ демонстрирует сглаженность $\mathrm{E}_{\mathrm{V}}$. Тогда все сечения $P / x=\{y: P(x, y)\}=f^{-1}(x)$ плоского борелевского множества $P=\{\langle x, y\rangle: f(y)=x\}-$ т.е. классы эквивалентности Витали - счетны. По классической теореме П. С. Новикова, $P$ допускает в этом случае представление $P=\bigcup_{n} P_{n}$, где все $P_{n}-$ униформные борелевские множества. Каждое из множеств $X_{n}=\left\{y: \exists x P_{n}(x, y)\right\}$ принадлежит $\Sigma_{1}^{1}$ (на самом деле даже борелевское), следовательно, измеримо по Лебегу, и $\mathbb{R}=\bigcup_{n} X_{n}$, благодаря чему хотя бы одно из $X_{n}$ имеет ненулевую меру. С другой стороны, $X_{n}$ имеет не более чем одну

\footnotetext{
${ }^{18} \mathrm{Cm} .[4]$ и [26] об истории вопроса.

${ }^{19}$ Smooth, в англоязычной литературе.
} 
общую точку с каждњм классом $\mathrm{E}_{\mathrm{V}}$-Эквивалентности, что быстро приводит $\mathrm{k}$ противоречию при помощи рассуждения Витали.

Примерно таким же способом можно доказать несглаженность аналога $\mathrm{E}_{\mathrm{V}}$ в $\mathcal{N}$, отношения

$$
\gamma \mathrm{E}_{0} \delta \longleftrightarrow \exists m \forall k \geqslant m[\gamma(k)=\delta(k)]
$$

на канторовом дисконтинууме $\mathscr{D}=2^{\omega}$, которое вообще играет принципиальную роль в классификации отношений эквивалентности.

4.2. Дихотомия Глимма-Эффроса. Точнее, мы увидим, что $\mathrm{E}_{0}$ является "наименьшим" среди несглаженных борелевских отношений - любое из них в известном смысле содержит $\mathrm{E}_{0}$.

ОПРЕДЕЛЕНИЕ 24. Пусть Е и $\mathrm{E}^{\prime}$ - отношения эквивалентности на $\mathscr{X}$ и $\mathscr{X}^{\prime}$, соответственно. Пишут $\mathrm{E} \leqslant \mathrm{E}^{\prime}$, когда сушествует борелевская функция $f: \mathscr{X} \rightarrow \mathscr{X}^{\prime}$ такая, что $x \mathrm{E} y \longleftrightarrow f(x) \mathrm{E}^{\prime} f(y)$ для всех $x, y \in \mathscr{X}$. (Такая функция $f$, если она существует,

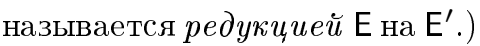

Пишут $\mathrm{E} \sqsubseteq \mathrm{E}^{\prime}$, когда сушествует взаимно однозначная (но не обязательно "на") борелевская функция с тем же свойством. (Такая функция, если она существует, на-

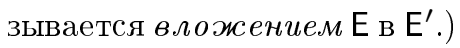

Понятно, что если в этой ситуации $\mathrm{E}^{\prime}$ - сглаженное отношение, то $\mathrm{E}$ - также сглаженное. Следовательно, любое отношение $\mathrm{E}$, удовлетворяющее $\mathrm{E}_{0} \sqsubseteq \mathrm{E}$, является несглаженным. Следующая теорема показьвает, что, более того, условие $\mathrm{E}_{0} \sqsubseteq \mathrm{E}$ является характеризующим для свойства несглаженности.

Теорема 25 (Харрингтон, Кекрис и Луво [12]). ${ }^{20}$ Пусть Е - борелевское отношение әквивалентности на $\mathcal{N}$. Тогда имеет место одно из двух:

(I) $\mathrm{E}$ - сглахсенное отношение;

(II) $\mathrm{E}_{0} \sqsubseteq \mathrm{E}$, причем даже посредством непрерьвной $f$.

Отсюда можно просто получить теорему Сильвера (теорема 19) в случае, когда $\mathrm{E}$ - борелевское, а не только ко-суслинское отношение. ${ }^{21}$ В самом деле, легко строится совершенное множество попарно $\mathrm{E}_{0}$-неэквивалентных точек; поэтому в случае (II) E также имеет совершенное множество попарно неэквивалентных точек. С другой стороны, дихотомия теоремы 19 для сглаженных борелевских отношений легко проверяется непосредственно.

Отметим, что как (I), так и (II) имеют несколько эквивалентных форм и дериватов, относяшихся к группам борелевских преобразований и теории меры, см. [12], а также [16].

Как и выше, теорема распространяется на все польские пространства.

\footnotetext{
${ }^{20}$ Даваемая этой теоремой дихотомия борелевских отношений эквивалентности известна под названием классификация Глимма-Eффроса, по имени математиков, впервые получивших результат для отношений класса $\mathbb{F}_{\sigma}$. См. статью [12] в связи с историей вопроса и различными приложениями в алгебре и теории вероятностей.

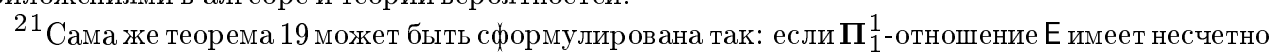
много классов эквивалентности, то $\Delta(\mathscr{D}) \sqsubseteq$ Е посредством непрерывной $f$, где $\Delta(\mathscr{D})$ - отношение равенства на канторовом дисконтинууме $\mathscr{D}=2^{\omega}$.
} 
ДокАЗАТЕльство. ${ }^{22}$ Итак, пусть Е - борелевское отношение эквивалентности на $\mathcal{N}$. Тогда Е принадлежит $\Delta_{1}^{1}(p)$ для некоторого $p \in \mathscr{N}$. Как обычно в таких случаях, мы проводим доказательство в предположении, что Е есть $\Delta_{1}^{1}$-отношение; в общем случае $p$ равномерно входит в рассуждения, так что скажем $\mathscr{T}$ меняется на $\mathscr{T}(p)$ и т. п.

Главной идеей доказательства является использование взаимоотношений между Е и $\overline{\mathrm{E}}$, замыканием $\mathrm{E}$ в топологии $\mathscr{T}^{2}$ (которая, напомним, есть произведение двух копий $\mathscr{T})$. Возможно одно из двух:

Случай 1: $\mathrm{E}=\overline{\mathrm{E}}$. т.е. Е замкнуто в $\mathscr{T}^{2}$.

Случай 2: $\mathrm{E} \varsubsetneqq \overline{\mathrm{E}}$.

Мы рассмотрим эти возможности по отдельности. Окажется, что в первом случае отношение $\mathrm{E}$ - сглаженное, а во втором случае $\mathrm{E}_{0}$ может быть непрерьвно вложено в $E$.

4.3. Случай замкнутого отношения. Итак, допустим, что $\mathrm{E}=\overline{\mathrm{E}}$.

Мы определяем $[A]_{\mathrm{E}}=\{x: \exists y \in A(x \mathrm{E} y)\}$ для $A \subseteq \mathscr{N}$ (Е-насыщение $\left.A\right)$. Множество $A$ называется Е-инвариантным, когда $A=[A]_{\mathrm{E}}$.

Следуюшая лемма понадобится для оценки дескриптивной сложности $\overline{\mathrm{E}}$.

Лемма 26. Если $\Sigma_{1}^{1}$-множества $A, B$ таковй, что $[A]_{\mathrm{E}} \cap[B]_{\mathrm{E}}=\varnothing$, то существует $\mathrm{E}$-инвариантное $\Delta_{1}^{1}$-множество $C$, отделяющее $[A]_{\mathrm{E}}$ от $[B]_{\mathrm{E}}$.

ДокАЗАТЕЛЬСТво. Используя то обстоятельство, что $[A]_{\mathrm{E}} \in \Sigma_{1}^{1}$ всякий раз, когда $A \in \Sigma_{1}^{1}$, и теорему отделимости (следствие 3 ), мы строим возрастающую последовательность множеств $A=C_{0} \subseteq C_{1} \subseteq C_{2} \subseteq \cdots$ так, что $C_{n}$ есть $\Delta_{1}^{1}$-множество, отделяюшее $\left[C_{n-1}\right]_{\mathrm{E}}$ от $[B]_{\mathrm{E}}$ при любом $n$. Объединение $C=\bigcup_{n} C_{n}$ Е-инвариантно и отделяет $[A]_{\mathrm{E}}$ от $[B]_{\mathrm{E}}$.

Сразу не видно, почему $C$ есть $\Delta_{1}^{1}$ (очевидно лишш, что $C$ - борелевское множество), однако более тонкий анализ (см. [12, лемма 5.1]) показывает, что теорема отделимости обладает равномерностью, достаточной для того, чтобы провести построение в варианте, гарантирующем $C \in \Delta_{1}^{1}$.

ЛЕмМа 27. $\overline{\mathrm{E}}$ - отношение әквивалентности класса $\Sigma_{1}^{1}$.

ДокАЗАТЕЛЬство. Согласно лемме 26, мы имеем по определению $\mathscr{T}$ :

$$
x \overline{\mathrm{E}} y \longleftrightarrow \forall C \in \Delta_{1}^{1}[C \quad \mathrm{E} \text {-инвариантно } \rightarrow(x \in C \rightarrow y \in C)]
$$

Следовательно, (счетное) семейство всех инвариантных $\Delta_{1}^{1}$-множеств $C$ является разделяющим. Но правая часть (5) приводится к $\Sigma_{1}^{1}$-виду таким же образом, как и в доказательстве леммы 20, при помощи принципа 4.

Итак, если $\mathrm{E}=\overline{\mathrm{E}}$, т.е. $\mathrm{E}$ замкнуто в $\mathscr{T}^{2}$, то $\mathrm{E}$ - сглаженное отношение.

4.4. Случай незамкнутого отношения. Продолжая доказательство теоремы 25 , мы теперь рассмотрим случай, когда $\mathrm{E} \varsubsetneqq \overline{\mathrm{E}}$, и докажем, что это предположение влечет $\mathrm{E}_{0} \sqsubseteq \mathrm{E}$.

\footnotetext{
${ }^{22}$ Мы следуем доказательству в [12]. Некоторые теоретико-рекурсивные факты удалось элиминировать. Конструкция, основанная в [12] на игре Шоке, заменена польскими сетями.
} 
Поскольку $\mathrm{E} \subseteq \overline{\mathrm{E}}$, каждый $\mathrm{E}$-класс $[x]_{\mathrm{E}}=\{y: x \mathrm{E} y\}$ включен в $\overline{\mathrm{E}}$-класс $[x]_{\overline{\mathrm{E}}}=$ $\{y: x \overline{\mathrm{E}} y\}$, причем по предположению имеются $\overline{\mathrm{E}}$-классы, содержащие более чем один Е-класс. Рассмотрим объединение

$$
H=\left\{x \in \mathscr{N}:[x]_{\mathrm{E}} \neq[x]_{\overline{\mathrm{E}}}\right\}=\{x: \exists y(x \overline{\mathrm{E}} y \& x \in \mathbb{E} y)\}
$$

всех таких классов; $H \in \Sigma_{1}^{1}$, поскольку $\mathrm{E} \in \Delta_{1}^{1}$ и $\overline{\mathrm{E}} \in \Sigma_{1}^{1}$. Множество $H$ играет здесь такую же ключевую роль, как и другое множество $H$ в доказательстве теоремы Сильвера 19 выше.

Лемма 28. В смысле топологии $\mathscr{T}^{2}$, Е плотно и первой категории в (открытом в $\overline{\mathrm{E}})$ множестве $H^{2} \cap \overline{\mathrm{E}}$.

ДокАЗАТЕЛЬСтво. Плотность очевидна; займемся проверкой утверждения о категории. Поскольку $\mathrm{E}$ - борелевское множество, предположение противного дает пару $\Sigma_{1}^{1}$-множеств $A, B \subseteq H$ таких, что $(A \times B) \cap \overline{\mathrm{E}} \neq \varnothing$, и $\mathrm{E}$ имеет вторую категорию (т.е. является множеством, дополнительным к множеству первой категории) на $(A \times B) \cap \overline{\mathrm{E}}$. Предполагаем, что $A \subseteq[B]_{\overline{\mathrm{E}}}$ и $B \subseteq[A]_{\mathrm{E}}$ (если это не так, то заменим $A$ на $A \cap[B]_{\overline{\mathrm{E}}}$, и то же для $\left.B\right)$.

Утверждается, что $A^{2} \cap \overline{\mathrm{E}} \subseteq \mathrm{E} ;$ другими словами, $\mathrm{E}$ и $\overline{\mathrm{E}}$ совпадают на $A$. Для доказательства рассмотрим множество $\overline{\mathrm{E}}^{3}=\{\langle x, y, z\rangle: x \overline{\mathrm{E}} y \overline{\mathrm{E}} z\}$ с топологией, унаследованной из $\mathscr{T}_{2+1}=\mathscr{T}_{2} \times \mathscr{T}$.

ФАКт 29. $\left\langle\overline{\mathrm{E}}^{3} ; \mathscr{T}_{2+1}\right\rangle$ имеет свойство бәровости.

ДокАЗАТЕЛьСтво. Достаточно указать польскую сеть для $\overline{\mathrm{E}}^{3}$ в топологии, унаследованной из $\mathscr{T}_{2+1}$. Для этого мы используем "генерические" польские сети $\left\{\mathscr{X}_{n}: n \in \omega\right\}$ и $\left\{\mathscr{Z}_{n}: n \in \omega\right\}$ топологий $\mathscr{T}_{2}$ и $\mathscr{T}$, даваемые леммой 9. Искомая сеть $\left\{\mathscr{P}_{n}: n \in \omega\right\}$ для $\overline{\mathrm{E}}^{3}$ задается следуюшим образом: $\mathscr{P}_{n}$ есть семейство всех непустых множеств вида $P=(X \times Z) \cap \overline{\mathrm{E}}^{3}$, где $X \in \mathscr{X}_{n}$ и $Z \in \mathscr{Z}_{n}$. Проверим требования определения 7 .

Плотность. Пусть $\Sigma_{1}^{1}$-множества $X \subseteq \mathscr{N}^{2}$ и $Z \subseteq \mathscr{N}$ таковы, что множество $P=(X \times Z) \cap \overline{\mathrm{E}}^{3}$ непусто. Возьмем произвольное $X^{\prime} \in \mathscr{X}_{n}$, удовлетворяюшее $X^{\prime} \subseteq X \cap\left([Z]_{\overline{\mathrm{E}}} \times[Z]_{\overline{\mathrm{E}}}\right)$, а затем произвольное $Z^{\prime} \in \mathscr{Z}_{n}$ такое, что $Z^{\prime} \subseteq X \cap\left[X^{\prime}\right]_{\overline{\mathrm{E}}}$; тогда $P^{\prime}=\left(X^{\prime} \times Z^{\prime}\right) \cap \overline{\mathrm{E}}^{3}$ принадлежит $\mathscr{P}_{n}$ и $P^{\prime} \subseteq P$.

Компактность. Пусть $P_{m}=\left(X_{m} \times Z_{m}\right) \cap \overline{\mathrm{E}}^{3} \in \mathscr{P}_{m}$ для каждого $m$ и все конечные пересечения непусты. Имеется единственная тройка $\langle x, y, z\rangle \in \mathscr{N}^{3}$ такая, что $\langle x, y\rangle \in \mathscr{X}_{n}$ и $z \in \mathscr{Z}_{n}$ для всех $n$. Проверим, что $\langle x, y, z\rangle \in \overline{\mathrm{E}}^{3}$. Пусть, напротив, скажем, $x \bar{E} z$. По лемме 26 существует Е-инвариантное $\Delta_{1}^{1}$-множество $C$, для которого $x \in C$ и $z \notin C$. Благодаря "генеричности" сетей $\left\{\mathscr{X}_{n}\right\}$ и $\left\{\mathscr{Z}_{n}\right\}$, найдутся числа $m, n$ такие, что $Z_{m} \cap C=\varnothing$ и $X_{n} \subseteq C \times \mathscr{N}$. Но в этом случае $P_{n} \cap P_{m}$ пусто - противоречие.

Продолжая доказательство включения $A^{2} \cap \overline{\mathrm{E}} \subseteq \mathrm{E}$, мы теперь рассмотрим $\mathscr{T}_{2+1}$-открытое в $\overline{\mathrm{E}}^{3}$ и непустое по предположению множество

$$
P=\left\{\langle x, y, z\rangle \in \overline{\mathrm{E}}^{3}: x \in A \& y \in A \& z \in B\right\} .
$$


По лемме 15 отображения $\overline{\mathrm{E}}^{3}$ в $\overline{\mathrm{E}}^{2}$, заданные посредством $\langle x, y, z\rangle \mapsto\langle x, z\rangle$ и $\langle x, y, z\rangle \mapsto$ $\langle y, z\rangle$ открыты, а значит, согласно выбору $A$ и $B$, множества $R=\{\langle x, y, z\rangle \in P: x \mathrm{E} z\}$ и $S=\{\langle x, y, z\rangle \in P: y \mathrm{E} z\}$ имеют $\mathscr{T}_{2+1}$-вторую категорию в $P$. Если теперь допустить противное, т.е. $A^{2} \cap \overline{\mathrm{E}} \not \mathbb{E}$, то множество $Q=\{\langle x, y, z\rangle \in P: x \not \mathbb{E} y\}$ окажется непустым. Но $Q$ открыто в $\mathscr{T}_{2+1}$, так как $P$ открыто, а $\mathrm{E} \in \Delta_{1}^{1}$. Поэтому $Q$ непусто пересекается с $R \cap S$ (мы используем бэровость топологии) - противоречие.

Итак, в самом деле, $\mathrm{E}$ совпадает с $\overline{\mathrm{E}}$ на $A$. Простое рассуждение показывает, что $[A]_{\mathrm{E}}=[A]_{\overline{\mathrm{E}}}$. (Иначе $\Sigma_{1}^{1}$-множество $A^{\prime}=[A]_{\overline{\mathrm{E}}} \backslash[A]_{\mathrm{E}}$ непусто, т.е. $\left(A^{\prime} \times A\right) \cap \overline{\mathrm{E}}$ также непусто. Тогда $\left(A^{\prime} \times A\right) \cap \mathrm{E} \neq \varnothing$, поскольку $\overline{\mathrm{E}}$ является замыканием $\mathrm{E}$-противоречие.) Но этого не может быть, так как $A \subseteq H$.

4.5. Вложение $\mathrm{E}_{0}$ в $\mathrm{E}$. Мы продолжаем доказательство теоремы (случай $\mathrm{E} \varsubsetneqq \overline{\mathrm{E}}$ ).

Согласно лемме 28 , имеется убываюшая последовательность $\mathscr{T}^{2}$-открытых в $\mathscr{N}^{2}$

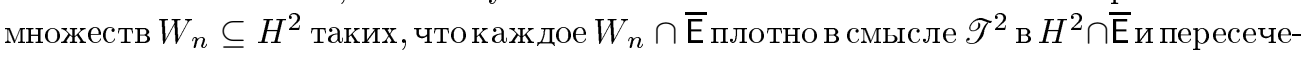
ние $\mathrm{E} \cap \bigcap_{n} W_{n}$ пусто. Можно предполагать, что диагональ $\Delta(H)=\{\langle x, x\rangle: x \in H\}$ дизъюнктна с $W_{0}$ (иначе заменим все $W_{n}$ разностями $W_{n} \backslash \Delta ; \Delta$ замкнуто даже в польской топологии).

Вложение $E_{0}$ в $E$, которое мы представим, основано на той же идее, что и классическое построение в статье Харрингтона, Кекриса и Луво [12]. Однако мы заменим игры Шоке (главньй инструмент обеспечения непустоты пересечений вдоль ветвей расщепления) польскими сетями, что несколько упрощает вькладки. Начнем с некоторых определений.

Пусть $P \subseteq \mathscr{N}^{2}$; положим $\operatorname{pr}_{1} P=\{x: \exists y P(x, y)\}$ и $\operatorname{pr}_{2} P=\{y: \exists x P(x, y)\}$.

Пусть $X, Y \subseteq \mathscr{N}$ и $\mathrm{R} \subseteq \mathscr{N}^{2}$. Мы пишем $X \mathrm{R} Y$, когда

$$
\forall x \in X \exists y \in Y(x \mathrm{R} y) \text { и } \forall y \in Y \exists x \in X(x \mathrm{R} y) .
$$

Согласно лемме 9 имеются польские сети $\left\{\mathscr{X}_{m}: m \in \omega\right\}$ и $\left\{\mathscr{P}_{m}: m \in \omega\right\}$ для топологий $\mathscr{T}$ на $\mathscr{N}$ и $\mathscr{T}_{2}$ на $\mathscr{N}^{2}$, соответственно. Мы положим

$$
\mathscr{X}_{n}^{*}=\left\{X: X \text { есть непустое } \Sigma_{1}^{1} \text {-множество и } \exists X^{\prime} \in \mathscr{X}_{n}\left(X \subseteq X^{\prime}\right)\right\}
$$

и аналогично определим $\mathscr{P}_{n}^{*}$ из $\mathscr{P}_{n}$.

Будет построено семейство $\Sigma_{1}^{1}$-множеств $X_{u}\left(u \in 2^{<\omega}\right)$ таких, что

(a) $X_{u} \in \mathscr{X}_{n-1}^{*}$ и $X_{u^{\wedge} i} \subseteq X_{u} \subseteq H$ для всех $n$ и всех $u \in 2^{n}$ и $i=0,1$.

(Мы напомним, что $2^{n}$ обозначает совокупность всех последовательностей 0 и 1 длины $n$, а $2^{<\omega}=\bigcup_{n \in \omega} 2^{n}$.) Отсюда следует, что для любого $a \in 2^{\omega}$ пересечение $\bigcap_{n \in \omega} X_{a \nmid n}$ содержит единственную точку, обозначаемую ниже через $\varphi(a)$, и отображение $\varphi$ непрерьвно в смысле польской топологии.

Чтобы обеспечить взаимную однозначность $\varphi$ и для некоторых других надобностей, необходимо еше одно требование - к парам $u, v \in 2^{<\omega}$.

(b) $X_{u} \times X_{v} \subseteq W_{n-1}$ для всех $n$ и каждой пары $u, v \in 2^{n}$ такой, что $u(n-1) \neq$ $v(n-1)$ (т.е. последние члены $u$ и $v$ различны). 
Заметим, что $\varphi(a) \neq \varphi(b)$ при $a \neq b \in 2^{\omega}$, поскольку $W_{0}$ дизъюнктно с $\Delta$; следовательно, $\varphi$ взаимно однозначна.

Нам потребуются дополнительные взаимосвязи между некоторыми парами $\langle u, v\rangle$, чтобы обеспечить правильное взаимодействие между ветвями в $2^{<\omega}$ и в конечном счете установить, что $\mathrm{E}_{0} \sqsubseteq \mathrm{E}$ посредством $\varphi$.

Пусть $u, v \in 2^{n}$. Скажем, что $u, v$ есть критическая пара, если $u=0^{k \wedge} 0^{\wedge} r$ и $v=0^{k \wedge} 1^{\wedge} r$ для некоторого $k<n\left(0^{k}\right.$ есть последовательность из $k$ нулей $)$ и какого-то $r \in 2^{n-k-1}$ (возможно, $k=n-1$, тогда $r=\Lambda$ ).

Таким образом, мы построим $\Sigma_{1}^{1}$-множества $\mathrm{R}_{u v}$ для всех критических пар $u, v$, так что будут выполнены следующие требования.

(c) $\operatorname{pr}_{1} \mathrm{R}_{u v}=X_{u}, \mathrm{pr}_{2} \mathrm{R}_{u v}=X_{v}, \mathrm{R}_{u^{\wedge} i, v} i \subseteq \mathrm{R}_{u v}$, каковы бы ни были критическая пара $u, v \in 2^{n}$ и $i \in\{0,1\}$.

(d) $\mathrm{R}_{u v} \in \mathscr{P}_{n-1}^{*}$ для любой критической пары $u, v \in 2^{n}$.

(е) Для каждого $k$ множество $\mathrm{R}_{k}=\mathrm{R}_{0^{k \wedge}} 0,0^{k \wedge} 1$ удовлетворяет $\mathrm{R}_{k} \subseteq \mathrm{E}$.

Заметим, что $u^{\wedge} i, v^{\wedge} i$ будет критической парой всякий раз, когда $u, v-$ критическая пара. Однако пара $u^{\wedge} i, v^{\wedge} j$ не может быть критической при $i \neq j$ (кроме случая $u=$ $v=0^{k}$ для некоторого $k$ ).

ЗАмечание 30 . Условие (с) влечет $X_{u} \mathrm{R}_{u v} X_{v}$, а тогда и $X_{u}$ Е $X_{v}$ согласно (е), для всех критических пар $u, v$. Поскольку любая пара $u, v \in 2^{n}$ может быть соединена конечной кепочкой критических пар в $2^{n}$, мы заключаем, что $X_{u} \mathrm{E} X_{v}$, следовательно, $X_{u} \overline{\mathrm{E}} X_{v}$ для все $x$ пар $u, v \in 2^{n}$.

Проверим, что условия (c)-(е) обеспечивают $\mathrm{E}_{0} \sqsubseteq \mathrm{E}$ посредством $\varphi$.

Докажем, что $a \mathrm{E}_{0} b$ влечет $\varphi(a) \mathrm{E} \varphi(b)$. Достаточно рассмотреть случай, когда $a=$ $0^{k \wedge} 0^{\wedge} c$ и $b=0^{k \wedge} 1^{\wedge} c$ для каких-то $c \in \omega$ и $k \in 2^{\omega}$, поскольку любая пара $u, v \in 2^{n}$ может быть связана в $2^{n}$ цепю критических пар. Пересечение $\bigcap_{n \in \omega} \mathrm{R}_{0^{k \wedge}} 0^{\wedge} c \nmid n, 0^{k \wedge} 1^{\wedge} c \nmid n$ непусто благодаря (d), но поскольку $\mathrm{R}_{u v} \subseteq X_{u} \times X_{v}$, пересечение не может быть чем-либо иным, кроме точки $\langle\varphi(a), \varphi(b)\rangle$. Значит, $\langle\varphi(a), \varphi(b)\rangle \in \mathrm{R}_{k}$, откуда $\varphi(a) \mathrm{E} \varphi(b)$ согласно (е).

Мы доказываем, что $a \mathbb{E}_{0} b$ влечет $\varphi(a) \mathbb{E} \varphi(b)$. В самом деле, $a \mathbb{E}_{0} b$ означает, что $a(n) \neq b(n)$ для бесконечно многих $n$; тогда $\langle\varphi(a), \varphi(b)\rangle \in W_{n}$ для бесконечно многих $n$ благодаря (b), т.е. фактически для всех $n$, так как множества $W_{n}$ убывают. Мы заключаем, что $\varphi(a)$ E $\varphi(b)$, ибо Е не имеет общих точек с пересечением множеств $W_{n}$.

Итак, для доказательства теоремы 25 остается построить множества $X_{u}$ и $\mathrm{R}_{u v}$, удовлетворяющие требованиям (a)-(e). Перед тем как начать собственно построение, докажем полезную комбинаторную лемму.

ЛЕмма 31. Пусть $n \in \omega u\left\{X_{u}: u \in 2^{n}\right\}$ - семейство непустых $\Sigma_{1}^{1}$-множеств. Пусть дополнительно для каждой критической пары $u, v \in 2^{n}$ задано $\Sigma_{1}^{1}$-множество $\mathrm{R}_{u v} \subseteq \mathscr{N}^{2}$, удовлетворяющее $X_{u} \mathrm{R}_{u v} X_{v}$.

1. Если $u_{0} \in 2^{n}$ u $X^{\prime} \subseteq X_{u_{0}}$ есть непустое $\Sigma_{1}^{1}$-множсество, то имеются непустые $\Sigma_{1}^{1}$-множества $Y_{u} \subseteq X_{u}\left(u \in 2^{n}\right)$ такие, что, по-прежснему, $Y_{u} \mathrm{R}_{u v} Y_{v}$ для всех критических пар $u, v$ и $Y_{u_{0}}=X^{\prime}$. 
2. Если $u_{0}, v_{0} \in 2^{n}$ - критическая пара, и непустые $\Sigma_{1}^{1}$-множества $X^{\prime} \subseteq$ $X_{u_{0}}$ и $X^{\prime \prime} \subseteq X_{v_{0}}$ удовлетворяют $X^{\prime} \mathrm{R}_{u_{0} v_{0}} X^{\prime \prime}$, то можно подобрать непустые $\Sigma_{1}^{1}$-множества $Y_{u} \subseteq X_{u}\left(u \in 2^{n}\right)$ такие, что, по-прежнему, $Y_{u} \mathrm{R}_{u v} Y_{v}$ для всех критических пар $u, v$ и $Y_{u_{0}}=X^{\prime}, Y_{v_{0}}=X^{\prime \prime}$.

ДокАЗАТЕЛЬСТво. Заметим, что 1 следует из 2: возьмем любое $v_{0}$ такое что одна из пар $\left\langle u_{0}, v_{0}\right\rangle,\left\langle v_{0}, u_{0}\right\rangle$ - критическая, и положим, соответственно, $X^{\prime \prime}=\left\{y \in X_{v_{0}}\right.$ : $\left.\exists x \in X^{\prime}\left(x \mathrm{R}_{u_{0} v_{0}} y\right)\right\}$ или $X^{\prime \prime}=\left\{y \in X_{v_{0}}: \exists x \in X^{\prime}\left(y \mathrm{R}_{v_{0} u_{0}} x\right)\right\}$.

Утверждение 2 доказывается индукцией по $n$.

Если $n=1$ - тогда $u_{0}=\langle 0\rangle$ и $v_{0}=\langle 1\rangle$ - положим $Y_{u_{0}}=Y^{\prime}$ и $Y_{v_{0}}=Y^{\prime \prime}$.

Шаг индукции. Мы доказываем лемму для $n+1$, предполагая, что она уже доказана для $n, n \geqslant 1$. Разделим множество $2^{n+1}$ на две части, $U_{0}=\left\{s^{\wedge} 0: s \in 2^{n}\right\}$ и $U_{1}=\left\{s^{\wedge} 1: s \in 2^{n}\right\}$, связанные единственной критической парой последовательностей $\widehat{u}=0^{n \wedge} 0$ и $\widehat{v}=0^{n \wedge} 1$.

Предположим, что $u_{0}=\widehat{u}$ и $v_{0}=\widehat{v}$. Применим индуктивное предположение (вариант 1) отдельно к системе $\left\{X_{u}: u \in U_{0}\right\}$ и множеству $X^{\prime} \subseteq X_{u_{0}}$ и к системе $\left\{X_{u}: u \in U_{1}\right\}$ и множеству $X^{\prime \prime} \subseteq X_{v_{0}}$. Соединив результаты вместе, получим систему $\Sigma_{1}^{1}$-множеств $Y_{u} \subseteq X_{u}\left(u \in 2^{n+1}\right)$ такую, что $Y_{u} \mathrm{R}_{u v} Y_{v}$ для всех критических пар $\langle u, v\rangle$, возможно, за исключением пары $u=u_{0}=\widehat{u}, v=v_{0}=\widehat{v}$, и дополнительно $Y_{u_{0}}=X^{\prime}$ и $Y_{v_{0}}=X^{\prime \prime}$. Наконец, отметим, что $Y_{\widehat{u}} \mathrm{R}_{\widehat{u} \widehat{v}} Y_{\widehat{v}}$ по выбору $X^{\prime}$ и $Y^{\prime}$.

Рассмотрим второй случай: $u_{0}$ и $v_{0}$ принадлежат одной и той же части, скажем, $U_{0}$. Применив индуктивное предположение (вариант 2) к системе $\left\{X_{u}: u \in U_{0}\right\}$ и множествам $X^{\prime} \subseteq X_{u_{0}}$ и $X^{\prime \prime} \subseteq X_{v_{0}}$, получим систему непустых $\Sigma_{1}^{1}$-множеств $Y_{u} \subseteq X_{u}\left(u \in U_{0}\right) ;$ в частности, $\Sigma_{1}^{1}$-множество $Y_{\widehat{u}} \subseteq X_{\widehat{u}}$. Теперь положим $Y_{\widehat{v}}=\left\{y \in X_{\widehat{v}}: \exists x \in Y_{\widehat{u}}\left(x \mathrm{R}_{\widehat{u} \widehat{v}} y\right)\right\}$, так что $Y_{\widehat{u}} \mathrm{R}_{\widehat{u} \widehat{v}} Y_{\widehat{v}}$, и применим индуктивное предположение (вариант 1 ) к семейству $\left\{X_{v}: v \in U_{1}\right\}$ и множеству $Y_{\widehat{v}} \subseteq X_{\widehat{v}}$.

4.6 Построение расщепляющейся системы. Начиная построение множеств $X_{u}$ и $\mathrm{R}_{u v}$, мы полагаем $X_{\Lambda}=H$.

Допустим, что система множеств $X_{s}\left(s \in 2^{n}\right)$ и отношений $\mathrm{R}_{s t}$ для критических пар $s, t \in 2^{k}, k \leqslant n$, уже определены; продолжим конструкцию на уровень $n+1$.

Сначала мы определим $A_{s^{\wedge} i}=X_{s}$ для всех $s \in 2^{n}$ и $i \in\{0,1\}$, а также $\mathrm{Q}_{u v}=\mathrm{R}_{s t}$ для каждой критической пары $u=s^{\wedge} i, v=t^{\wedge} i$ в $2^{n+1}$, кроме пары $\widehat{u}=0^{n \wedge} 0, \widehat{v}=$ $0^{n \wedge} 1$; для последней (заметим, что $A_{\widehat{u}}=A_{\widehat{v}}=X_{0^{n}}$ ) определим $\mathrm{Q}_{\widehat{u} \widehat{v}}=\overline{\mathrm{E}}$, так что $A_{u} \mathrm{Q}_{u v} A_{v}$ выполнено для всех критических пар $u, v \in 2^{n+1}$.

Множества $A_{u}$ и $\mathrm{Q}_{u v}$ будут уменьшены в несколько приемов, чтобы удовлетворить условия (а)-(e).

После $2^{n+1}$ шагов применения леммы 31 (вариант 1 ) имеем систему непустых $\Sigma_{1}^{1}$-множеств $B_{u} \subseteq A_{u}, B_{u} \in \mathscr{X}_{n}^{*}\left(u \in 2^{n+1}\right)$, выполняюших $B_{u} \mathrm{Q}_{u v} B_{v}$ для каждой критической пары $u, v$ в $2^{n+1}$. Это обеспечивает (a).

Чтобы гарантировать (b), рассмотрим любую пару $u_{0}=s_{0}^{\wedge} 0, v_{0}=t_{0}^{\wedge} 1$, где $s_{0}, t_{0} \in 2^{n}$. Согласно замечанию 30 и $\mathscr{T}^{2}$-плотности $\mathscr{T}^{2}$-открытого множества $W_{n}$ в $H^{2} \cap \overline{\mathrm{E}}$, сушествуют непустые $\Sigma_{1}^{1}$-множества $B^{\prime} \subseteq B_{u_{0}}$ и $B^{\prime \prime} \subseteq B_{v_{0}}$ такие, что $B^{\prime} \times B^{\prime \prime} \subseteq W_{n}$ и $P=\left(B^{\prime} \times B^{\prime \prime}\right) \cap \overline{\mathrm{E}}$ непусто. Можно предположить, что $\operatorname{pr}_{1} P=B^{\prime}$ и $\operatorname{pr}_{2} P=B^{\prime \prime}$ (если это не так, положим $B^{\prime}=\operatorname{pr}_{1} P$ и $B^{\prime \prime}=\operatorname{pr}_{2} P$ ). 
В этом предположении $B^{\prime} \overline{\mathrm{E}} B^{\prime \prime}$. Теперь применим лемму 31 (вариант 1) отдельно к семействам $\left\{B_{s^{\wedge} 0}: s \in 2^{n}\right\}$ и $\left\{B_{t^{\wedge} 1}: t \in 2^{n}\right\}$ (ср. с доказательством леммы 31 ) и множествам $B^{\prime} \subseteq B_{s_{0}} 0, B^{\prime \prime} \subseteq B_{t_{0} 1}$, соответственно. Соединив результаты, мы получим систему непустых $\Sigma_{1}^{1}$-множеств $B_{u}^{\prime} \subseteq B_{u}\left(u \in 2^{n+1}\right)$ таких, что $B_{u_{0}}^{\prime}=B^{\prime}$, $B_{v_{0}}^{\prime}=B^{\prime \prime}$, т.е. $B_{u_{0}}^{\prime} \times B_{v_{0}}^{\prime} \subseteq W_{n}$, и всё еще $B_{u}^{\prime} \mathrm{Q}_{u v} B_{v}^{\prime}$ для всех критических пар $u, v$ в $2^{n+1}$, возможно, за исключением пары $\widehat{u}=0^{n \wedge} 0, \widehat{v}=0^{n \wedge} 1$, единственной соединяющей две области. Для этой особой пары, заметим, что $B_{\widehat{u}}^{\prime} \overline{\mathrm{E}} B_{u_{0}}^{\prime}$ и $B_{\widehat{v}}^{\prime} \overline{\mathrm{E}} B_{v_{0}}^{\prime}$ (замечание 30 работает в каждой из двух областей), так что $B_{\widehat{u}}^{\prime} \overline{\mathrm{E}} B_{\widehat{v}}^{\prime}$, поскольку $B^{\prime} \overline{\mathrm{E}} B^{\prime \prime}$. Наконец, на данный момент $\mathrm{Q}_{\widehat{u} \widehat{v}}$ есть $\overline{\mathrm{E}}$ по определению, откуда следует, что $B_{\widehat{u}}^{\prime} \mathrm{Q}_{\widehat{u} \widehat{v}} B_{\widehat{v}}^{\prime}$.

После $2^{n+1}$ шагов (число пар $u_{0}, v_{0}$, которые нужно рассмотреть) мы получаем систему непустых $\Sigma_{1}^{1}$-множеств $C_{u} \subseteq B_{u}\left(u \in 2^{n+1}\right)$ такую, что $C_{u} \times C_{v} \subseteq W_{n}$ всякий раз, когда $u(n) \neq v(n)$, а также $C_{u} \mathrm{Q}_{u v} C_{v}$ для всех критических пар $u, v \in 2^{n+1}$. Теперь с требованием (b) все в порядке.

Обеспечим (е) для пары $\widehat{u}=0^{n \wedge} 0, \widehat{v}=0^{n \wedge} 1$. На данный момент, $\mathrm{Q}_{\widehat{u} \widehat{v}}=\overline{\mathrm{E}}$. Используя то, что $\mathrm{E} \mathscr{T}^{2}$-плотно в $\overline{\mathrm{E}}_{\text {и }} C_{\widehat{u}} \overline{\mathrm{E}} C_{\widehat{v}}$, мы находим, что множество $\mathrm{Q}=\left(C_{\widehat{u}} \times C_{\widehat{v}}\right) \cap \mathrm{E}$ непусто. Рассмотрим $\Sigma_{1}^{1}$-множества $C^{\prime}=\operatorname{pr}_{1} \mathrm{Q}\left(\subseteq C_{\widehat{u}}\right)$ и $C^{\prime \prime}=\operatorname{pr}_{2} \mathrm{Q}\left(\subseteq C_{\widehat{v}}\right)$; ясно, что $C^{\prime} \mathrm{Q} C^{\prime \prime}$, откуда $C^{\prime} \mathrm{Q}_{\widehat{u} \widehat{v}} C^{\prime \prime}$. Лемма 31 (вариант 2) приносит систему непустых $\Sigma_{1}^{1}$-множеств $D_{u} \subseteq C_{u}\left(u \in 2^{n+1}\right)$ такую, что все еще $D_{u} \mathrm{Q}_{u v} D_{v}$ для всех критических пар $u, v$ в $2^{n+1}$ и $D_{\widehat{u}}=C^{\prime}, D_{\widehat{v}}=C^{\prime \prime}$. Мы переопределяем $\mathrm{Q}_{\widehat{u} \widehat{v}}$ посредством $\mathrm{Q}_{\widehat{u} \widehat{v}}=\mathrm{Q}$; соотношение $D_{\widehat{u}} \mathrm{Q}_{\widehat{u} \widehat{v}} D_{\widehat{v}}$ при этом сохраняется.

Наконец, обеспечим (c) и (d). Рассмотрим произвольную критическую пару $u_{0}=s_{0}^{\wedge} 0, v_{0}=t_{0}^{\wedge} 1$ в $2^{n+1}$. Множество $\mathrm{Q}^{\prime}=\mathrm{Q}_{u_{0}} v_{0} \cap\left(D_{u_{0}} \times D_{v_{0}}\right)$ есть непустое (так как $D_{u_{0}} \mathrm{Q}_{u_{0} v_{0}} D_{v_{0}}$ ) $\Sigma_{1}^{1}$-подмножество $\mathrm{Q}_{u_{0} v_{0}}$. Возьмем любое непустое $\Sigma_{1}^{1}$-множество $\mathrm{Q} \subseteq \mathrm{Q}^{\prime}$, принадлежашее $\mathscr{P}_{n}^{*}$. Пусть $D^{\prime}=\operatorname{pr}_{1} \mathrm{Q}$ и $D^{\prime \prime}=\mathrm{pr}_{2} \mathrm{Q}$ (тогда $D^{\prime} \mathrm{Q} D^{\prime \prime}$, поскольку $D^{\prime} \mathrm{Q}_{u_{0} v_{0}} D^{\prime \prime}$ ). Применим лемму 31 (вариант 2) к системе множеств $D_{u}\left(u \in 2^{n+1}\right)$ и множествам $D^{\prime}$ и $D^{\prime \prime}$. После этого вводим “новое" $\mathrm{Q}_{u_{0} v_{0}}$ через $\mathrm{Q}_{u_{0} v_{0}}=\mathrm{Q}$.

Проделаем это последовательно для всех критических пар; множества, полученные в конце концов, - обозначим их через $X_{u}\left(u \in 2^{n+1}\right)$ - являются искомыми. Отношения $\mathrm{R}_{u v}\left(u, v \in 2^{n+1}\right)$ получаются сужением множеств $\mathrm{Q}_{u v}$ на $X_{u} \times X_{v}$.

Этим построение завершено.

Доказательство теоремы 25 закончено.

4.7. Некоторые другие результаты. (Это краткое представление не претендует на полноту, но направлено на демонстрацию богатства идей в этой области дескриптивной теории.)

Согласно теореме 19 , равенство $\Delta(\mathscr{D})$ на канторовом дисконтинууме $\mathscr{D}=2^{\omega}$ является 巨-наименшшим среди борелевских отношений, имеюших несчетно много классов эквивалентности. Оставив в стороне сглаженные отношения, мы видим, что Е $\mathrm{E}_{0}$ 巨-наименьшее среди несглаженных борелевских отношений. Одна из главных проблем в этой области - достичь понимания того, что происходит вьше $\mathrm{E}_{0}$.

Гиперконечные и гиперсглаженные отношения.

Дохерти, Джексон и Кекрис [11] рассмотрели важный частньй случай әиперконечныц отношений, т.е. отношений вида $\mathrm{E}=\bigcup_{n} \mathrm{E}_{n}$, где $\mathrm{E}_{n} \subseteq \mathrm{E}_{n+1}$ и каждое $\mathrm{E}_{n}$ 
- конечное отношение (это значит, что каждый класс эквивалентности $\mathrm{E}_{n}-$ конечное множество). Борелевские гиперконечные отношения - это в точности те, которые индуцированы действием борелевского автоморфизма. Для таких отношений в статье [11] показано следующее. Во-первых, каждое несглаженное отношение Е этого вида подобно $\mathrm{E}_{0}$ в том смысле, что $\mathrm{E} \sqsubseteq \mathrm{E}_{0}$ и $\mathrm{E}_{0} \sqsubseteq \mathrm{E}$. (Второе следует из теоремы 25.) Во-вторых, получена более тонкая классификация борелевских несглаженных гиперконечных отношений с точностью до борелевской изоморфности: указано счетное множество различаемых типов изоморфизма.

Второй важный класс борелевских отношений-это гиперсглаженные отношения, т.е. те, которые имеют вид $\mathrm{E}=\bigcup_{n} \mathrm{E}_{n}$, где $\mathrm{E}_{n} \subseteq \mathrm{E}_{n+1}$ и все $\mathrm{E}_{n}$ - сглаженные. Кекрис и Луво [17] нашли еше одну теорему о дихотомии: для любого борелевского гиперсглаженного отношения $\mathrm{E}$ либо $\mathrm{E} \leqslant \mathrm{E}_{0}$, либо $\mathrm{E}_{1} \leqslant \mathrm{E}$, где $\mathrm{E}_{1}$ - отношение на пространстве $\mathscr{D}^{\omega}$ всех бесконечных последовательностей точек $\mathscr{D}=2^{\omega}$, определенное через

$$
\left\langle x_{n}\right\rangle_{n \in \omega} \mathrm{E}_{1}\left\langle y_{n}\right\rangle_{n \in \omega} \longleftrightarrow \exists n \forall m \geqslant n\left(x_{m}=y_{m}\right),
$$

а порядок $\leqslant$ (он слабее чем $\sqsubseteq$ ) введен определением 24 . Отсюда удалось вывести результат, которьй показьвает, что для порядка $\leqslant$ теоремы 19 и 25 в принципе исчерпывают все предложения этого типа. Назовем борелевское отношение R критическим, если оно $\leqslant$-сравнимо с любым иным борелевским отношением эквивалентности Е. Оказывается, что отношения равенства $\Delta(\mathscr{D}), \Delta(\omega)$ и $\Delta(\{1,2, \ldots, n\}), n \in \omega$, исчерпьвают (с точностью до $\mathrm{R} \approx \mathrm{R}^{\prime}$, когда $\mathrm{R} \leqslant \mathrm{R}^{\prime}$ и $\mathrm{R}^{\prime} \leqslant \mathrm{R}$ ) все критические борелевские отношения. Отсюда следует, что парами дихотомического типа среди борелевских отношений являются только:

(1) $\left\langle\Delta(\mathscr{D}), \mathrm{E}_{0}\right\rangle$ - теорема 25;

(2) $\langle\Delta(\omega), \Delta(\mathscr{D})\rangle$ - теорема Сильвера;

(3) $\langle\Delta(n), \Delta(n+1)\rangle$ для всех $n-$ тривиально.

Отношения класса $\boldsymbol{\Sigma}_{1}^{1}$.

Подобно исследованиям в связи с теоремой Сильвера (о чем мы уже говорили в конще раздела 3), вызывает большой интерес проблема обобщения теоремы 25 на отношения эквивалентности класса $\boldsymbol{\Sigma}_{1}^{1}$.

Непосредственно теорема 25 для $\boldsymbol{\Sigma}_{1}^{1}$-отношений не проходит. Действительно, пусть E - отношение, введенное в конце раздела 3 ; в частности, E - тонкое, т.е. не допускает совершенного множества попарно неэквивалентных точек, но имеет несчетно много классов эквивалентности. Тогда $\mathrm{E}_{0} \sqsubseteq \mathrm{E}$ невозможно, поскольку существует совершенное множество попарно $\mathrm{E}_{0}$-неэквивалентных точек; в равной мере $\mathrm{E}$ - не сглаженное, так как тонкое сглаженное отношение может иметь лишь счетно много классов.

Совсем недавно Хъёрт и Кекрис [15] показали, что суть проблемы заключается в неадекватности определения сглаженности в ситуации, когда рассматриваются $\boldsymbol{\Sigma}_{1}^{1}$-отношения.

Назовем отношение $\mathrm{E}$ сглажсенным по Ульм $_{\text {ль }}{ }^{23}$, если существует $\boldsymbol{\Delta}_{1}$-функция ${ }^{24}$

\footnotetext{
${ }^{23}$ Хъёрт и Кекрис дают ссылку на теорему Ульма о классификации счетных абелевых $p$-групп, которую можно интерпретировать в данном контексте.

${ }^{24} \mathrm{C}$ точки зрения сложности, класс $\boldsymbol{\Delta}_{1}$ примерно равносилен проективному $\boldsymbol{\Delta}_{2}^{1}$, но определяется в рамках теоретико-множественной, а не дескриптивной иерархии.
} 
$f: \mathcal{N} \rightarrow 2^{<\omega_{1}}$ такая, что $x \mathrm{E} y \longleftrightarrow f(x)=f(y)$ для всех $x, y \in \mathscr{N}$. (Заметим, что $2^{<\omega_{1}}$ есть множество всех счетных - любой длины $<\omega_{1}$ - бинарных последовательностей.) Легко видеть, что каждое сглаженное в рассмотренном вьше смысле отношение будет и сглаженным по Ульму.

В работе [15] показано, что если все классы $\Sigma_{1}^{1}$-отношения Е суть борелевские множества, то либо $\mathrm{E}_{0} \sqsubseteq \mathrm{E}$, либо $\mathrm{E}$ - сглаженное по Ульму.

Полностью избежать неприятного требования борелевости классов пока не удается. Хъёрт и Кекрис [15] доказали альтернативу: $\mathrm{E}_{0} \sqsubseteq$ Е либо сглаженность по Ульму для произвольного $\Sigma_{1}^{1}$-отношения $\mathrm{E}$ в одном предположении, связанном с сушествованием измеримого кардинала. Нет никаких оснований считать, что столь сильная гипотеза здесь на самом деле нужна, хотя бы потому что до сих пор мы не знаем ни одного контрпримера. ${ }^{25}$

\section{5. Расщепление плоских борелевских множеств}

Наше последнее приложение топологии, порожденной эффективно суслинскими множествами, связано с интересной и важной темой: плоские борелевские множества со специальными сечениями.

5.1. Теорема о расщеплении. Рассмотрим множество $P \subseteq \mathscr{N}^{2}=\mathscr{N} \times \mathscr{N}$; подмножества “бэровской плоскости” $\mathscr{N}^{2}$ уместно называть плоскими множествами. Каждая точка $x \in \mathscr{N}$ определяет сечение

$$
P / x=\{y:\langle x, y\rangle \in P\}
$$

множества $P .{ }^{26}$ Допустим, что все сечения $P / x$ суть множества класса $\Sigma_{\lambda}^{0}$, где $2 \leqslant$ $\lambda<\omega_{1}-$ фиксированный ординал. Тогда по аксиоме выбора мы имеем $P=\bigcup_{n} P_{n}$, где каждое $P_{n}$ обладает тем свойством, что все сечения $P_{n} / x(x \in \mathscr{N})$ принадлежат классу $\boldsymbol{\Pi}_{<\lambda}^{0}=\bigcup_{1 \leqslant \xi<\lambda} \Pi_{\xi}^{0}$.

Допустим теперь, что $P$ - борелевское множество; можно ли добиться того, чтобы множества $P_{n}$ также были борелевскими? Классическая дескриптивная теория дала в некоторых случаях положительный ответ на подобные вопросы. Например, если каждое из сечений $P / x$ борелевского $P$ не более чем счетно, то $P=\bigcup P_{n}$, где каждое $P_{n}$ - борелевское униформное множество. Если каждое сечение $P / x \sigma$-компактно, то можно требовать, чтобы все сечения $P_{n} / x$ были компактными, а все множества $P_{n}-$ борелевскими. (Теоремы П. С. Новикова, см. [4].)

Теорема 32 (Луво [18]). Пусть $2 \leqslant \lambda<\omega_{1}$ и борелевское $P \subseteq \mathscr{N} \times \mathscr{N}$ таково, что $P / x \in \boldsymbol{\Sigma}_{\lambda}^{0}$ для любого $x \in \mathcal{N}$. Найдется последовательность борелевских множеств $P_{n}$ такая, что $P=\bigcup_{n} P_{n}$ и для любых п и $x P_{n} / x$ есть множество из $\Pi_{<\lambda}^{0}$.

\footnotetext{
${ }^{25}$ Недавно автором установлено, что эта альтернатива выполняется во всех генерических расширениях конструктивного универсума.

${ }^{26}$ Мы используем это обозначение $P / x$, чтобы избежать коллизий с индексами.
} 
ДокАЗАТЕЛЬСтво. Перед тем как обратиться к техническим деталям, кратко изложим идею доказательства. Построение борелевского множества $X \subseteq \mathscr{N}$ можно формализовать в виде счетной последовательности операций счетного объединения и дополнения, начиная с бэровских интервалов; будем называть это борелевским построением. Каждое борелевское построение имеет определенную длину - ординал $\lambda<\omega_{1}$, показываюший число элементарных шагов в построении и дающий таким образом верхнюю границу борелевского класса результирующего множества $X$.

Может, однако, случиться (к этому, например, мы сразу приходим в анализе теоремы 32), что фактически борелевское $X$ имеет класс много ниже, чем тот, который формально вытекает из длины данного построения $X$. Таким образом, мы можем иметь построение П длины $\lambda$ для $X$ и знать, что на самом деле существует построение $\Pi^{\prime}$ известной длины $\xi<\lambda$.

Оказывается, и это главный момент в доказательстве теоремы, что в этом случае имеется в некотором смысле эффективная процедура, как "короткое" построение $\Pi^{\prime}$ может быть найдено исходя из П. Например, в условиях теоремы мы сможем эффективно сопоставить каждому $x$ борелевское построение сечения $P / x$ именно в виде $\Sigma_{\lambda}^{0}$-множества, что предусматривает автоматическое представление $P / x$ в виде счетного объединения $\Pi_{<\lambda}^{0}$-множеств, "интегрируя" которые по $x$, мы и получим теорему.

Теперь перейдем к деталям. Мы начинаем с группы важных определений.

5.2. Кодировка борелевских множеств. Предполагается фиксированной нумерация $\{\mathscr{N}(n): n \in \omega\}$ всех бэровских интервалов $\mathscr{N} ;$ например, $\mathscr{N}(n)=B_{n}[\mathscr{N}]$ в смысле раздела 1.3 .

Пусть $x \in \mathscr{N}$ и $n \in \omega$. Мы определяем $(x)_{n} \in \mathscr{N}$ равенством $(x)_{n}(k)=x(\ulcorner n, k\urcorner+1)$ для всех $k$, где, напомним, $\ulcorner n, k\urcorner=2^{n}(2 k+1)-1$. Таким образом, каждой совокупности из числа $m \in \omega$ и набора точек $x_{n} \in \mathscr{N}(n \in \omega)$ взаимно однозначно соответствует точка $x \in \mathscr{N}$ такая, что $x(0)=m$ и $(x)_{n}=x_{n}$ для всех $n$.

Наконец, для $x \in \mathscr{N}$ мы определим $x^{-} \in \mathscr{N}$ условием $x^{-}(k)=x(k+1)$ для всех $k$. Отображение $x \mapsto\left\langle x(0), x^{-}\right\rangle$есть биекция $\mathscr{N}$ на $\omega \times \mathscr{N}$.

Теперь мы вводим совокупность борелевских кодов $\mathrm{BC} \subseteq \mathscr{N}$ и для каждого $k \in \mathrm{BC}$ - (борелевское) множество $\mathbb{B}(c) \subseteq \mathscr{N}$. Именно, ВС есть наименьшее подмножество $\mathscr{N}$ такое, что

(1) ВС содержит все $c \in \mathscr{N}$, удовлетворяюшие $c(0)=0$. - Для каждого такого с ми полагаем $\mathbb{B}(c)=\bigcup_{c^{-}(c)=1} \mathscr{N}(c)$.

(2) Если $c(0)=1$ и $c^{-} \in \mathrm{BC}$, то $c \in \mathrm{BC}$. - Если в этом случае $\mathbb{B}\left(c^{-}\right)$уже определено, то полагаем $\mathbb{B}(c)=\mathscr{N} \backslash \mathbb{B}\left(c^{-}\right)$.

(3) Если $c(0)=2$ и $(c)_{n} \in \mathrm{BC}$ для всех $n$, то $c \in \mathrm{BC} .-$ Если при этом все множества $\mathbb{B}\left((c)_{n}\right)$ уже определень, то полагаем $\mathbb{B}(c)=\bigcup_{n} \mathbb{B}\left((c)_{n}\right)$.

Легко видеть, что $\{\mathbb{B}(c): c \in \mathrm{BC}\}$ есть семейство всех борелевских подмножеств $\mathscr{N}$. Индукцией по $\lambda, 1 \leqslant \lambda<\omega_{1}$, мы определяем множества кодов $\sigma_{\lambda} \subseteq \mathrm{BC}$ и $\pi_{\lambda} \subseteq \mathrm{BC}$, порождаюшие конкретные борелевские классы:

1. $\boldsymbol{\sigma}_{1}=\{c \in \mathscr{N}: c(0)=0\}$;

2. при любом $\lambda, \boldsymbol{\pi}_{\lambda}=\left\{c: c(0)=1 \& c^{-} \in \boldsymbol{\sigma}_{\lambda}\right\} \cup \boldsymbol{\pi}_{<\lambda} \cup \boldsymbol{\sigma}_{<\lambda}$;

3. при $\lambda>1, \boldsymbol{\sigma}_{\lambda}=\left\{c: c(0)=2 \& \forall n(c)_{n} \in \boldsymbol{\pi}_{<\lambda}\right\} \cup \boldsymbol{\pi}_{<\lambda} \cup \boldsymbol{\sigma}_{<\lambda}$. 
(Здесь и далее $\boldsymbol{\pi}_{<\lambda}=\bigcup_{1 \leqslant \xi<\lambda} \boldsymbol{\pi}_{\xi}$ и т. п. для аналогичных обозначений.) Таким образом, $\left\{\mathbb{B}(c): c \in \boldsymbol{\sigma}_{\lambda}\right\}$ есть совокупность всех $\boldsymbol{\Sigma}_{\lambda}^{0}$-множеств $X \subseteq \mathscr{N}$, и аналогично для пары $\boldsymbol{\pi}_{\lambda}$ и $\boldsymbol{\Pi}_{\lambda}^{0}$.

Принцип 33 ([24], см. также [1, гл. 20] или [2]). Множсества ВС, $\{\langle c, x\rangle: c \in \mathrm{BC}$ $\& x \in \mathbb{B}(c)\} u\{\langle c, x\rangle: c \in \mathrm{BC} \& x \notin \mathbb{B}(c)\}$ принадлежат классу $\Pi_{1}^{1}$.

ОПРЕДЕЛЕНИЕ 34 [эффективные борелевские коды и множества]. Пусть $p \in \mathcal{N}$. Мы полагаем $\sigma_{\lambda}(p)=\sigma_{\lambda} \cap \Delta_{1}^{1}(p), \pi_{\lambda}(p)=\pi_{\lambda} \cap \Delta_{1}^{1}(p)$ и

$$
\Sigma_{\lambda}^{0}(p)=\left\{\mathbb{B}(c): c \in \sigma_{\lambda}(p)\right\}, \quad \Pi_{\lambda}^{0}(p)=\left\{\mathbb{B}(c): c \in \pi_{\lambda}(p)\right\}
$$

Как обычно, в случае, когда $p$ отсутствует, $\sigma_{\lambda}=\sigma_{\lambda} \cap \Delta_{1}^{1}, \pi_{\lambda}=\pi_{\lambda} \cap \Delta_{1}^{1}$ и $\Sigma_{\lambda}^{0}=$ $\left\{\mathbb{B}(c): c \in \sigma_{\lambda}\right\}, \Pi_{\lambda}^{0}=\left\{\mathbb{B}(c): c \in \pi_{\lambda}\right\}$.

Таким образом, определены эффективные подклассы $Г$ и $\Gamma(p)^{27}$ в каждом из классов $\boldsymbol{\Gamma}=\boldsymbol{\Sigma}_{\lambda}^{0}$ и $\boldsymbol{\Pi}_{\lambda}^{0}$. Множества, скажем, класса $\Sigma_{\lambda}^{0}$ суть в точности те $\boldsymbol{\Sigma}_{\lambda}^{0}$-множества, которые допускают эффективное (в смысле: принадлежащее $\Delta_{1}^{1}$ ) построение из бэровских интервалов.

5.3. Эффективный вариант теоремы Луво. Согласно принципу 33 , любое, скажем, $\Sigma_{\lambda}^{0}(p)$-множество принадлежит как $\boldsymbol{\Sigma}_{\lambda}^{0}$, так и $\Delta_{1}^{1}(p)$. Очень важно, что при некоторых необременительных условиях имеет место и обратное включение.

Теорема 35 (Луво [18]). Допустим, что $p \in \mathscr{N}$ u $\lambda<\omega_{1}$ таковы, что множества $\boldsymbol{\sigma}_{\xi}, \boldsymbol{\pi}_{\xi}, \boldsymbol{\sigma}_{<\xi}, \boldsymbol{\pi}_{<\xi}$, где $\xi \leqslant \lambda$, все принадлежсат $\Delta_{1}^{1}(p)$. Тогда каждое мнохество $X \subseteq \mathscr{N}$ из $\Sigma_{\lambda}^{0} \cap \Delta_{1}^{1}(p)$ принадлежит $\Sigma_{\lambda}^{0}(p)$.

Этим доказывается то, о чем мы неформально говорили выше: эффективное множество класса $\Sigma_{\lambda}^{0}$ әффективно принадлежит этому классу.

Отметим, что на самом деле эта теорема несколько ослаблена в нашем изложении по сравнению со статьей Луво [18]. Во-первых, Луво доказьвает теорему отделимости, которая здесь могла бы быть сформулирована так: если в условиях теоремы 35 дизъюнктные $\Sigma_{1}^{1}(p)$-множества $X$ и $Y$ таковы, что первое $\boldsymbol{\Pi}_{\lambda}^{0}$-отделимо от второго, то имеется отделяющее множество в классе $\Pi_{\lambda}^{0}(p)$. Во-вторых, взаимосвязь между $p$ и $\lambda$ в [18] вьглядит так: $\lambda$ - рекурсивньй ординал относительно $p$ (тогда можно доказать, что все множества, упомянутые в теореме 35 , в самом деле принадлежат $\left.\Delta_{1}^{1}(p)\right)$.

Вьвод теоремы 32 из теоремы 35. Зафиксируем $p_{0} \in \mathscr{N}$ такое, что данное множество $P$ и все множества $\boldsymbol{\sigma}_{\xi}, \boldsymbol{\pi}_{\xi}, \boldsymbol{\sigma}_{<\xi}, \boldsymbol{\pi}_{<\xi}$, где $1 \leqslant \xi \leqslant \lambda$, принадлежат $\Delta_{1}^{1}\left(p_{0}\right)$. Для $x \in \mathscr{N}$ пусть $p_{x} \in \mathscr{N}$ определено посредством равенств $p_{x}(2 k)=p_{0}(k), p_{x}(2 k+1)=$ $x(k)$ для всех $k$. Тогда каждое сечение $P / x$ принадлежит $\Sigma_{\lambda}^{0}$, следовательно, $\Sigma_{\lambda}^{0}\left(p_{x}\right)$

\footnotetext{
${ }^{27} \mathrm{~B}$ частном случае $\lambda=1$, мы имеем маленькую неприятность: в самом деле, классы $\Sigma_{1}^{0}(p)$ и $\Pi_{1}^{0}(p)$ уже были определены в разделе 1 , и можно проверить, что данное здесь определение вводит значительно более широкие классы, чем данное в разделе 1 . Чтобы разрешить эту трудность, мы просто забудем в этом разделе об определении классов $\Gamma_{1}^{0}(p)$ из раздела 1.
} 
по теореме 35 . Это означает, что найдется код $c \in \sigma_{\lambda}\left(p_{x}\right)=\sigma_{\lambda} \cap \Delta_{1}^{1}\left(p_{x}\right)$ такой, что $P / x=\mathbb{B}(c)$. Мы заключаем, что множество

$$
U=\left\{\langle x, k\rangle: k \in \sigma_{\lambda}\left(p_{x}\right) \& \mathbb{B}(c)=P / x\right\}
$$

удовлетворяет $\operatorname{dom} U=\mathscr{N}$. Однако $U \in \Pi_{1}^{1}\left(p_{0}\right)$ (принцип 33 и следствие 6 ). Значит, по теореме униформизации (теорема 1$)$ найдется $\Pi_{1}^{1}\left(p_{0}\right)$-функция $F$ такая, что $U(x, F(x))$ для всех $x \in \mathscr{N}$.

Заметим, что на самом деле $F$ принадлежит даже $\Delta_{1}^{1}\left(p_{0}\right)$, так как

$$
F(x)=c \longleftrightarrow \forall c^{\prime} \in \Delta_{1}^{1}\left(p_{x}\right)\left(c \neq c^{\prime} \rightarrow F(x) \neq c\right),
$$

и можно использовать следствие 5 . В частности, $F-$ борелевская функция и $F(x) \in$ $\sigma_{\lambda}, \mathbb{B}(F(x))=P / x$ для любого $x$.

Согласно определению $\sigma_{\lambda}$, в этом случае можно задать счетную последовательность борелевских функций $F_{n}(n \in \omega)$ так, чтобы $F_{n}(x) \in \boldsymbol{\pi}_{<\lambda}$ и $P / x=\mathbb{B}(F(x))=$ $\bigcup_{n \in \omega} \mathbb{B}\left(F_{n}(x)\right)$ для всех $x$. Теперь остается положить $P_{n}=\left\{\langle x, y\rangle: y \in \mathbb{B}\left(F_{n}(x)\right)\right\}$.

5.4. Доказательство эффективной теоремы. Прежде всего, договоримся доказьвать теорему 35 в случае, когда $p$ отсутствует, т.е. для классов $\Delta_{1}^{1}$ и $\Sigma_{\lambda}^{0}$; см. замечание в конце раздела 2.1 .

Мы используем индукцию по $\lambda$. Уже в случае $\lambda=1$ (база индукции) нужна некоторая изобретательность. Пусть $X \subseteq \mathscr{N}$ открыто и $\Delta_{1}^{1}$; докажем, что $X \in \Sigma_{1}^{0} \cdot{ }^{28}$ Будет достаточно найти $\Delta_{1}^{1}$-множество $N \subseteq \omega$ такое, что $X=\bigcup_{n \in N} \mathscr{N}(n)$; получить код $c \in \sigma_{1}$, удовлетворяюший $X=\mathbb{B}(c)$, из такого $N$ не составляет труда.

Положим $\mathrm{K}=\{n \in \omega: \mathscr{N}(n) \subseteq X\}$; тогда $X=\bigcup_{n \in K} \mathscr{N}(n)$, однако непосредственно мы имеем только $K \in \Pi_{1}^{1}$. Чтобы заменить $K \Delta_{1}^{1}$-множеством с таким же свойством, используется рассуждение, на которое мы будем ссылаться ниже как на $(\Pi \rightarrow \Delta)$-прием. Униформизуя $\Pi_{1}^{1}$-множество

$$
P=\{\langle x, n\rangle: x \in X \& x \subseteq \mathscr{N}(n) \subseteq X\}
$$

при помоши следствия 2 , мы имеем $\Delta_{1}^{1}$-функцию выбора $Q$, сопоставляюшую каждому $x \in X$ число $n=Q(x) \in \omega$ такое, что $x \in \mathcal{N}(n) \subseteq X$. Тогда множество $L=\{n: \exists x(n=Q(x))\}$ удовлетворяет $X=\bigcup_{n \in L} \mathscr{N}(n)$. С другой стороны, $L \subseteq K$ и $L$ есть $\Sigma_{1}^{1}$. По теореме отделимости (следствие 3 ) сушествует $\Delta_{1}^{1}$-множество $N$, $L \subseteq N \subseteq K$. Это $N$ и является искомым.

Индуктивный шаг. Предполагаем, что $2 \leqslant \lambda<\omega_{1}$ и теорема 35 уже доказана ниже $\lambda$, т.е. $\Sigma_{\xi}^{0} \cap \Delta_{1}^{1} \subseteq \Sigma_{\xi}^{0}$ при $\xi<\lambda$. Идея доказательства для самого $\lambda$ состоит в использовании того же метода, что и для случая $\lambda=1$. Роль бэровских интервалов будут играть множества из $\Pi_{<\lambda}^{0}$. Следуюшая лемма показывает наличие определенных предпосылок для такого подхода.

\footnotetext{
${ }^{28}$ См. сноску 27 . Теперь $\Sigma_{1}^{0}$-множества - это открытые множества с $\Delta_{1}^{1}$-кодом.
} 
Лемма 36. Пусть $\xi \leqslant \lambda u A \subseteq \sigma_{\xi}$ есть $\Delta_{1}^{1}$-множество, причем если $\xi=\lambda$, то $A \subseteq \sigma_{<\lambda} \cup \pi_{<\lambda}$. Тогда $X=\bigcup_{a \in A} \mathbb{B}\left(\right.$ a) принадлежит $\Sigma_{\xi}^{0}$.

ДокАЗАТЕльство. Во-первых, мы видим, что $X \in \Delta_{1}^{1}$, поскольку

$$
\text { [для } \left.\Pi_{1}^{1}\right] \quad x \in X \longleftrightarrow \exists a \in \Delta_{1}^{1}[a \in A \& x \in \mathbb{B}(a)]
$$

и

$$
\text { [для } \left.\Sigma_{1}^{1}\right] \quad x \in X \longleftrightarrow \exists a[a \in A \& \neg(x \notin \mathbb{B}(a))]
$$

(использованы следствие 5 и принцип 33). Индуктивное предположение теоремы сразу закрьвает случай $\xi<\lambda$. Остается рассмотреть случай $\xi=\lambda$, когда код для $X$ должен быть дан прямым построением.

Необходимо найти $\Delta_{1}^{1}$-перечисление элементов $A$. Для этого мы заметим, что принцип 4 (часть 2) в частном случае, когда $p=\omega \times\{0\}$ (тождественньй ноль), приносит множество $K \subseteq \omega$ и точку $\mathbf{d}_{n} \in \mathcal{N}$ для всех $n \in K$, так что $\Delta_{1}^{1} \cap \mathscr{N}=\left\{\mathbf{d}_{n}: n \in K\right\}$ и множества

$$
K, \quad\left\{\left\langle n, \mathbf{d}_{n}\right\rangle: n \in K\right\}, \quad\left\{\langle n, x\rangle: n \in K \& x \neq \mathbf{d}_{n}\right\}
$$

принадлежат $\Pi_{1}^{1}$. Теперь обратимся к $(\Pi \rightarrow \Delta)$-приему.

Множество $P=\left\{\langle a, n\rangle: a \in A \& n \in K \& \mathbf{d}_{n}=a\right\}$ также принадлежит $\Pi_{1}^{1}$; при этом $\operatorname{dom} P=A$ есть $\Delta_{1}^{1}$-множество. Согласно следствию 2 , сушествует униформизуюшее $\Delta_{1}^{1}$-множество $Q \subseteq P$. Тогда $L=\{n: \exists a Q(a, n)\}$ есть $\Sigma_{1}^{1}$-подмножество $K$, т.е. благодаря отделимости найдется $\Delta_{1}^{1}$-множество $N, L \subseteq N \subseteq K$.

Имеется два принципиальных момента. Во-первых, $A=\left\{\mathbf{d}_{n}: n \in N\right\}$. Во-вторых, $F=\left\{\left\langle n, \mathbf{d}_{n}\right\rangle: n \in N\right\}$ принадлежит $\Delta_{1}^{1}$, по вьшесказанному. Второе обстоятельство показьвает, что код $a \in \mathrm{BC}$, определенньй так, что $a(0)=1,\left(a^{-}\right)_{n}=\mathbf{d}_{n}$ для $n \in N$, и $\left(a^{-}\right)_{n}=e$ (где $e-$ фиксированный простой код пустого множества) при $n \notin N,-$ принадлежит $\Delta_{1}^{1}$, а значит, $\sigma_{\lambda}$. Благодаря первому обстоятельству, $\mathbb{B}(a)=X$.

Здесь будет уместно дать доказательство еще одной леммы, основанное на $(\Pi \rightarrow \Delta)$-приеме. Значение этой леммы состоит в том, что пересечения над $\Pi_{1}^{1}$-множествами индексов могут быть в определенных случаях аппроксимированы пересечениями над $\Delta_{1}^{1}$-множествами.

Лемма 37. Пусть $A \subseteq \mathrm{BC} \cap \Delta_{1}^{1}$ есть $\Pi_{1}^{1}$-множество, причем $X=\bigcap_{a \in A} \mathbb{B}(a)$ не пересекается с $\Sigma_{1}^{1}$-множеством $Z$. Найдется $\Delta_{1}^{1}$-множество $A^{\prime} \subseteq A$ такое, что $X^{\prime}=\bigcap_{a \in A^{\prime}} \mathbb{B}($ a) также не пересекает $Z$.

ДоказАтельство. Множество $P=\{\langle x, c\rangle: c \in A \& x \notin \mathbb{B}(c)\}$ принадлежит $\Pi_{1}^{1}$ благодаря принципу 33. Следовательно, по теореме униформизации (принцип 1) найдется $\Pi_{1}^{1}$-множество $Q \subseteq P$, униформизуюшее $P$. Множество $A^{\prime \prime}=$ $\{c: \exists x \in Z Q(x, c)\}$ есть $\Sigma_{1}^{1}$; действительно,

$$
c \in A^{\prime \prime} \longleftrightarrow \exists x \in Z \forall c^{\prime} \in \Delta_{1}^{1}\left[c^{\prime} \neq c \rightarrow \neg Q\left(x, c^{\prime}\right)\right]
$$

и остается воспользоваться следствием 5. Теорема отделимости (принцип 3) дает $\Delta_{1}^{1}$-множество $A^{\prime}$ такое, что $A^{\prime \prime} \subseteq A^{\prime} \subseteq A$. Тогда $X^{\prime}=\bigcap_{c \in A^{\prime}} \mathbb{B}(c)$ не имеет 
общих точек с $Z$. (Если $x \in Z$, то найдется $c$ такое, что $Q(x, c)$; тогда $c \in A^{\prime \prime}$, и, следовательно, $c \in A^{\prime}$. Но по выбору $Q$ и определению $P$ будет $x \notin \mathbb{B}(c)$, т.е. $x \notin X^{\prime}$.)

Продолжая доказательство теоремы 35 , мы введем для $\xi \leqslant \lambda \Sigma_{\xi}^{*}$ как семейство всех множеств вида $Y=\bigcup_{a \in A} \mathbb{B}(a)$, где $A \subseteq \sigma_{\xi}$ есть $\Pi_{1}^{1}$-множество. Аналогично, $\Pi_{\xi}^{*}$ обозначает семейство всех множеств $\bigcap_{a \in A} \mathbb{B}(a)$, где $A \subseteq \pi_{\xi}$ опять принадлежит классу $\Pi_{1}^{1}$.

$\Phi \mathrm{AKT} 38 . \Sigma_{\xi}^{*} \subseteq \Pi_{1}^{1} u \Pi_{\xi}^{*} \subseteq \Sigma_{1}^{1}$.

ДокАЗАТЕЛьСтво. См. начало доказательства леммы 36.

После этих приготовлений мы докажем лемму, которая обеспечит индуктивный шаг теоремы 35. В известном смысле индуктивный шаг теоремы просто редуцирован к индуктивному шагу леммы.

ЛЕмМа 39. Если $2 \leqslant \rho \leqslant \lambda$, то каждое $\Pi_{\rho}^{0}$-множество $\mathscr{T}$-почти равно 29 некоторому (счетному) пересечению множеств класса $\Sigma_{<\rho}^{*}=\bigcup_{1 \leqslant \xi<\rho} \Sigma_{\xi}^{*}$.

ДокАЗАтЕльство. Используем индукцию по $\rho$. Итак, пусть $2 \leqslant \rho \leqslant \lambda$.

Рассмотрим $\boldsymbol{\Pi}_{\rho}^{0}$-множество $X \subseteq \mathscr{N} . X$ есть счетное пересечение множеств из $\boldsymbol{\Sigma}_{<\rho}^{0}$, т.е. можно считать, что само $X$ принадлежит $\boldsymbol{\Sigma}_{\xi}^{0}$ для какого-то $\xi<\rho$. По индуктивному предположению, $X$ T-почти равно счетному объединению множеств из $\Pi_{<\xi}^{*}$. Значит, можно допустить, что $X$ и есть счетное объединение множеств из $\Pi_{<\xi}^{*}$ (или бэровских интервалов - при $\xi=1)$.

Обозначим через $X^{*}$ пересечение всех $\Sigma_{\xi}^{*}$-множеств, содержаших $X$. Поскольку $\Sigma_{\xi}^{*}$ - счетный класс, для окончания доказательства леммы будет достаточно проверить, что разность $X^{*} \backslash X$ имеет $\mathscr{T}$-первую категорию. Фактически мы докажем, что $X^{*} \backslash X \quad \mathscr{T}$-нигде не плотно.

Итак, для произвольного непустого $\Sigma_{1}^{1}$-множества $Z$ мы найдем непустое $\Sigma_{1}^{1}$-множество $Z^{\prime} \subseteq Z$, не имеющее общих точек с $X^{*} \backslash X$.

Случай 1. $Z \cap X$ непусто. Тогда, по предположению об $X$, существует $\Pi_{<\xi^{\text {-мно- }}}$ жество (или бэровский интервал - при $\xi=1) Y \subseteq X$, также непусто пересекающееся с $Z$. Однако $Y$ есть $\Sigma_{1}^{1}$ (факт 38$)$. Остается взять $Z^{\prime}=Z \cap Y$.

Случай 2. $Z \cap X=\varnothing$. Мы покажем, что тогда $Z \cap X^{*}=\varnothing$, т.е. можно взять просто $Z^{\prime}=Z$. Сначала рассмотрим подслучай, когда $\xi>1$. Согласно леммам 37 и 36

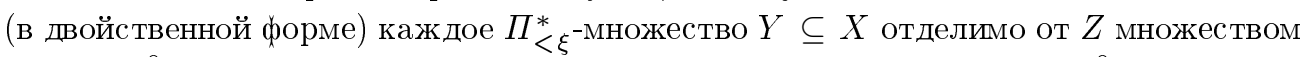

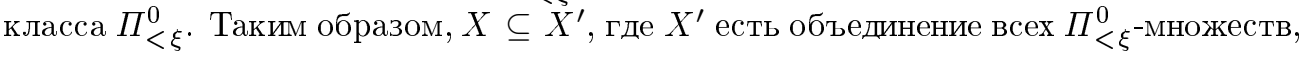
не пересекающихся с $Z$.

Покажем, что $X^{\prime} \in \Sigma_{\xi}^{*}$; тогда $X^{*} \subseteq X^{\prime}$, и, далее, $X^{*} \cap Z=\varnothing$. Достаточно проверить, что множество $C=\left\{c \in \pi_{<\xi}: \mathbb{B}(c) \cap Z=\varnothing\right\}$ принадлежит $\Pi_{1}^{1}$. Имеем

$$
c \in C \longleftrightarrow c \in \Delta_{1}^{1} \& c \in \pi_{<\xi} \& \forall z(z \notin Z \vee z \notin \mathbb{B}(c))
$$

\footnotetext{
${ }^{29}$ Два множества $X$ и $Y \tau$-почти равны, где $\tau$ - топология, если симметрическая разность $X \triangle Y$ есть множество первой категории в смысле $\tau$.
} 
Отношение $c \in \Delta_{1}^{1}$ выражается $\Pi_{1}^{1}$-формулой $\exists a \in \Delta_{1}^{1}(c=a)$ (мы ссылаемся на следствие 5). Второй конъюнктивньй член есть $\Pi_{1}^{1}$ согласно условию теоремы. Наконец, последний член имеет тот же класс по выбору $Z$ и принципу 33.

Теперь рассмотрим подслучай, когда $\xi=1$. Здесь роль множеств из $\Pi_{<1}^{*}$ и $\Pi_{<1}^{0}$ играют бэровские интервалы. Таким образом, пусть $X^{\prime}$ есть объединение всех бэровских интервалов, не пересекающихся с $Z$. Требуется проверить, что $X^{\prime} \in \Sigma_{1}^{*}$. Напомним, что $\mathcal{N}(n)$ обозначает $n$-й бэровский интервал в смысле некоторого эффективного перечисления этих интервалов. Мы имеем $X^{\prime}=\bigcup_{n \in N} \mathcal{N}(n)$, где $N=\{n: \mathscr{N}(n) \cap Z=\varnothing\}$ есть $\Pi_{1}^{1}$-множество.

Определим $c_{n} \in \mathscr{N}$ условиями $c_{n}(0)=0, c_{n}^{-}(n)=1$ и $c_{n}^{-}(k)=0$ при $k \neq n$, так что $\mathbb{B}\left(c_{n}\right)=\mathscr{N}(n)$. Тогда $C=\left\{c_{n}: n \in N\right\}$ принадлежит $\Pi_{1}^{1}$ вместе с $N$. С другой стороны, $X^{\prime}=\bigcup_{c \in C} \mathbb{B}(c)$, т.е. $X^{\prime} \in \Sigma_{1}^{*}$.

Мы возвращаемся к доказательству теоремы 35 (индуктивньй шаг). Рассмотрим произвольное множество $X \subseteq \mathscr{N}$ из $\Pi_{\lambda}^{0} \cap \Delta_{1}^{1}$ и докажем, что $X$ принадлежит $\Pi_{\lambda}^{0}$. Согласно лемме, $X \quad \mathscr{T}$-почти равно счетному пересечению множеств из $\Sigma_{<\lambda}^{*}$. Пусть $U$ - одно из этих $\Sigma_{<\lambda}^{*}$-множеств, т.е. разность $D=X \backslash U$ есть множество $\mathscr{T}$-первой категории. Но $U \in \Pi_{1}^{1}$ (факт 38 ), а значит, $D \in \Sigma_{1}^{1}$. Напомним, что $\mathscr{T}-$ бэровская топология по лемме 13 , т.е. открытое множество первой категории может быть только пустым. Значит, фактически, $X \subseteq U$. Используя леммы 37 и 36 , мы видим, что существует $\Sigma_{<\lambda}^{0}$-множество $V$ такое, что $X \subseteq V \subseteq U$.

Вывод: пересечение $X^{*}$ всех $\Sigma_{<\lambda}^{0}$-множеств $V \supseteq X$ T-почти равно $X$. Мы имеем $X^{*}=\bigcap_{a \in A} \mathbb{B}(a)$, где $A=\left\{a \in \sigma_{<\lambda}: X \subseteq \mathbb{B}(a)\right\}$. Легко проверяется, что $A \in \Pi_{1}^{1}$, т.е. $X^{*} \in \Pi_{<\lambda}^{*}$ и $X^{*} \in \Sigma_{1}^{1}$ (факт 38 ). Лемма 13 дает $X=X^{*}$. Применяя лемму $37 \mathrm{k}$ $X^{*}$ и дополнению $X$, а затем лемму 36 , мы окончательно имеем $X \in \Pi_{\lambda}^{0}$.

5.5. Заключительное замечание. Теорема 35 выражает, несомненно, факт более фундаментального характера, чем теорема 32 , хотя последняя выглядит более классической. Вот еще одно приложение.

СЛЕДСТВИЕ 40. Если $1 \leqslant \lambda<\omega_{1}$, то множество $\sigma_{\lambda}^{\prime}=\left\{c \in \mathrm{BC}: \mathbb{B}(c) \in \Sigma_{\lambda}^{0}\right\}$ борелевских кодов $\boldsymbol{\Sigma}_{\lambda}^{0}$-множеств принадлежст $\boldsymbol{\Pi}_{1}^{1}$.

ДокАЗАТЕльство. Выберем $p_{0}$ и определим $p_{x}$, как в рассуждении сразу после формулировки теоремы 35 (только без требования, относящегося к $P$ ). При любом $c \in \mathrm{BC}$ множество $\mathbb{B}(c)$ принадлежит $\Delta_{1}^{1}(c)$ согласно принципу 33 . Следовательно, если $\mathbb{B}(c) \in \Sigma_{\lambda}^{0}$, то по теореме $35 \mathbb{B}(c)=\mathbb{B}\left(c^{\prime}\right)$ для некоторого $c^{\prime} \in \sigma_{\lambda}\left(p_{c}\right)=\sigma_{\lambda} \cap \Delta_{1}^{1}\left(p_{c}\right)$. Значит,

$$
c \in \boldsymbol{\sigma}_{\lambda}^{\prime} \longleftrightarrow \exists c^{\prime} \in \Delta_{1}^{1}\left(p_{c}\right)\left(c^{\prime} \in \boldsymbol{\sigma}_{\lambda} \& \mathbb{B}(c)=\mathbb{B}\left(c^{\prime}\right)\right) .
$$

Равенство $\mathbb{B}(c)=\mathbb{B}\left(c^{\prime}\right)$ можно выразить $\Pi_{1}^{1}$-отношением, используя принцип 33 ; затем остается воспользоваться следствием 5 .

Прямая проверка дает только $\boldsymbol{\sigma}_{\lambda}^{\prime} \in \boldsymbol{\Sigma}_{2}^{1}$, что, конечно, много хуже; и даже используя такое мощное средство, как теорема Мартина о борелевской детерминированности, удается вывести лишш $\boldsymbol{\sigma}_{\lambda}^{\prime} \in \boldsymbol{\Delta}_{2}^{1}$ - это демонстрирует силу теоремы 35 . В качестве одного из приложений результатов типа следствия 40 (речь идет о его эффективном 
варианте в том же смысле, как теорема 35 может быть названа эффективным вариантом теоремы 32) мы упомянем один из результатов статьи [3]: если среди конституант $\Sigma_{1}^{1}$-множества, заданного посредством борелевского решета, несчетно много непустых (для $\boldsymbol{\Sigma}_{1}^{1}$-множеств это не влечет неборелевость!), то эти конституанты не образуют семейство ограниченного борелевского ранга.

\section{СПИСОК ЛИТЕРАТУРЫ}

[1] Й Иех Т. Теория множеств и метод форсинга. М.: Мир, 1973.

[2] Кановей В.Г. Проективная иерархия Н. Н. Лузина: современное состояние теории // Справочная книга по математической логике. Часть II. Теория множеств. М.: Наука, 1982. C. $273-364$.

[3] Кановей В. Г. Разрешимые и неразрешимые свойства конституант // Матем. сб. 1984. Т. 124 (166). № 4 . С. 505-535.

[4] Кановей В. Г. Развитие дескриптивной теории множеств под влиянием трудов Н.Н. Лузина // УМН. 1985. Т. 40. №3. С. 117-155.

[5] Мартин Д. А. Дескриптивная теория множеств: проективные множества // Справочная книга по математической логике. Часть II. Теория множеств. М.: Наука, 1982. С. 235-272.

[6] Тихомиров В.М. Открытие А-множеств // Историко-математические исследования. Вып. 34. М.: Наука, 1993. С. 129-139.

[7] Успенский В.А., Кановей В.Г. Вклад М.Я. Суслина в теоретико-множественную математику // Вестн. МГУ. Сер. 1. Матем., мех. 1988. № 5. С. 22-30.

[8] Шенфилд Дж. Математическая логика. М.: Наука, 1975.

[9] Alexandroff P. Sur la puissance des ensembles mesurables (B) // C. R. Acad. Sci. Paris. 1916. V. 162. Р. 323-325; Александров П.С. Теория функций действительного переменного и теория топологических пространств. М.: Наука, 1978. С. 35-39.

[10] Burgess J. Effective enumeration of classes in a $\Sigma_{1}^{1}$ equivalence relation // Indiana Univ. Math. J. 1979. V. 28. P. 353-364.

[11] Dougherty R., Jackson S., Kechris A.S. The structure of hyperfinite Borel equivalence relations // Trans. Amer. Math. Soc. 1994. V. 341. № 1. P. 193-225.

[12] Harrington L. A., Kechris A.S., Louveau A. A Glimm-Effros dichotomy for Borel equivalence relations // J. Amer. Math. Soc. 1990. V. 3. № 4. P. 903-928.

[13] Hausdorff F. Die Mächtigkeit der Borelschen Mengen // Math. Ann. 1916. V. 77. № 3. P. 430-437.

[14] Hjorth G. Thin equivalence relations and effective decompositions // J. Symbolic Logic. 1993. V. 58. № 4. P. 1153-1164.

[15] Hjorth G., Kechris A.S. Analytic equivalence relations and Ulm-type classification // J. Symbolic Logic. 1996. V. 60 (to appear).

[16] Kechris A.S. Topology and descriptive set theory // Topology Appl. 1994. V. 58. № 3. P. 195-222.

[17] Kechris A.S., Louveau A. The classification of hypersmooth Borel equivalence relations // Preprint.

[18] Louveau A. A separation theorem for $\Sigma_{1}^{1}$ sets // Trans. Amer. Math. Soc. 1980. V. 260. P. 363-378.

[19] Mansfield R., Weitkamp G. Recursive aspects of descriptive set theory (Oxford Logic Guides: II). Oxford: Clarendon Press, 1985.

[20] Martin D. A., Kechris A.S. Infinite games and effective descriptive set theory // Analytic Sets / ed. C. A. Rogers et al. New York-London: Academic Press, 1980. P. 403-470.

[21] Miller A. W. Descriptive set theory and forcing: how to prove theorems about Borel sets the hard way // University of Wisconsin Madison, January, 1994. 
[22] Sami R. L. On $\Sigma_{1}^{1}$ equivalence relations with Borel classes of bounded rank // J. Symbolic Logic. 1984. V. 49. № 4. P. 1273-1283.

[23] Silver J. Counting the number of equivalence classes of Borel and coanalytic equivalence relations // Ann. Math. Logic. 1980. V. 18. P. 1-28.

[24] Solovay R. M. A model of set theory in which every set of reals is Lebesgue measurable // Ann. Math. 1970. V. 92. № 1. P. 1-56.

[25] Souslin M. Sur une définition des ensembles mesurables B sans nombres transfinis // C. R. Acad. Sci. Paris. 1917. V. 164. P. 88-90.

[26] Stern J. On Lusin's restricted continuum problem // Ann. Math. 1984. V. 120. P. 7-37.

Московский государственный

университет путей сообщения (МГУПС-МИИТ)

E-mail: kanovei@mech.math.msu.su, kanovei@math.uni-wuppertal.de
Поступила в редакцию

29.06 .1995 